\title{
Finite $N$ corrections to the superconformal index of orbifold quiver gauge theories
}

\author{
Reona Arai, Shota Fujiwara, Yosuke Imamura and Tatsuya Mori \\ Department of Physics, Tokyo Institute of Technology, \\ Tokyo 152-8551, Japan \\ E-mail: r.arai@th.phys.titech.ac.jp, s.fujiwara@th.phys.titech.ac.jp, \\ imamura@phys.titech.ac.jp, t.mori@th.phys.titech.ac.jp
}

ABSTRACT: We investigate the AdS/CFT correspondence for quiver gauge theories realized on D3-branes put on abelian orbifolds by using the superconformal index. We assume that on the gravity side the finite $N$ corrections of the index are reproduced by D3-branes wrapped on three particular three-cycles in the internal space $\mathcal{Y}$, the abelian orbifold of $\boldsymbol{S}^{5}$. We first establish the relation between baryonic charges on the gauge theory side and the D3-brane wrapping numbers and holonomies on D3-branes. Then we confirm our proposal by comparing the results of localization for gauge theories and the results on the AdS side including the contributions of D3-branes and excitation on them for many examples. We only focus on the leading finite $N$ corrections starting from $q^{N}$, and leave the sub-leading corrections starting at $q^{k N}(k \geq 2)$ as a task for the future. We find complete agreement for the leading corrections in all examples.

KEYwORDS: AdS-CFT Correspondence, D-branes, Supersymmetric Gauge Theory

ARXIV EPRINT: 1907.05660 


\section{Contents}

1 Introduction 1

2 Orbifolds and quiver gauge theories 4

2.1 Toric diagrams 4

2.2 Quiver gauge theories 5

$\begin{array}{lll}2.3 \text { Baryonic charges } & 7\end{array}$

3 Large $N$ limit $\quad 9$

3.1 Gravity multiplet $\quad 9$

$\begin{array}{ll}3.2 & \text { Tensor multiplet } \\ \end{array}$

4 Finite $N$ corrections from wrapped D3-branes $\quad 11$

4.1 Wrapping number 11

$\begin{array}{ll}4.2 \text { Wrapped D3-branes and baryonic charges } & 12\end{array}$

$\begin{array}{lll}4.3 & \text { Index from wrapped D3-branes } & 15\end{array}$

5 Examples $\quad \mathbf{1 8}$

$5.1 \mathbb{C}^{3} / \mathbb{Z}_{2 n+1}(n=1,2,3, \ldots) \quad 18$

$\begin{array}{lll}5.1 .1 & \mathbb{C}^{3} / \mathbb{Z}_{3} & 18\end{array}$

$\begin{array}{lll}5.1 .2 & \mathbb{C}^{3} / \mathbb{Z}_{5} & 20\end{array}$

$\begin{array}{lll}5.1 .3 & \mathbb{C}^{3} / \mathbb{Z}_{7} & 22\end{array}$

5.2 The other $\mathbb{C}^{3} / \mathbb{Z}_{7} \quad 23$

$5.3 \mathbb{C} \times \mathbb{C}^{2} / \mathbb{Z}_{n}(n=2,3,4, \ldots) \quad 25$

$\begin{array}{lll}5.3 .1 & \mathbb{C} \times \mathbb{C}^{2} / \mathbb{Z}_{2} & 25\end{array}$

$\begin{array}{lll}5.3 .2 & \mathbb{C} \times \mathbb{C}^{2} / \mathbb{Z}_{3} & 27\end{array}$

$5.4 \mathbb{C}^{3} / \mathbb{Z}_{4} \quad 28$

$\begin{array}{lll}5.5 & \mathbb{C}^{3} /\left(\mathbb{Z}_{2} \times \mathbb{Z}_{2}\right) & 30\end{array}$

$\begin{array}{lll}6 & \text { Summary and discussions } & 32\end{array}$

A Results for $N=3 \quad 33$

A.1 $\mathbb{C}^{3} / \mathbb{Z}_{3}$

A.2 $\mathbb{C}^{3} / \mathbb{Z}_{5}$

A.3 $\mathbb{C} \times \mathbb{C}^{2} / \mathbb{Z}_{2}$

A.4 $\mathbb{C} \times \mathbb{C}^{2} / \mathbb{Z}_{3}$

A.5 $\mathbb{C}^{3} / \mathbb{Z}_{4} \quad 37$

A.6 $\mathbb{C}^{3} /\left(\mathbb{Z}_{2} \times \mathbb{Z}_{2}\right)$ 


\section{Introduction}

An $\mathcal{N}=1$ gauge theory is realized on the worldvolume of $N$ D3-branes put at the apex of a Calabi-Yau cone $\mathcal{X}$. If $N$ is sufficiently large the D3-brane system is well described as the classical supergravity solution $\operatorname{AdS}_{5} \times \mathcal{Y}$, where $\mathcal{Y}$ is the base of the cone $\mathcal{X}$. The AdS/CFT correspondence [1-3] claims that the type IIB string theory in this background is dual to the superconformal gauge theory realized on the D3-branes at IR. This duality has been tested by calculating various quantities on the both sides and confirming their agreement. In this paper we focus on the superconformal index [4]. We define the index as a formal power series of $q^{\frac{1}{2}}$ with the coefficient of each term being a Laurent polynomial of other fugacities. A BPS operator with the dimension $d$ and the right-handed spin $\bar{j}$ contributes $\propto q^{d+\bar{j}}$ to the index. See (1.4) for an explicit definition.

In the large $N$ limit the agreement of the superconformal index has been confirmed in a large class of the internal spaces $\mathcal{Y}$ and the corresponding superconformal field theories. For $\mathcal{N}=4 \mathrm{U}(N)$ SYM corresponding to $\mathcal{Y}=\boldsymbol{S}^{5}$, the large $N$ index was calculated on the both sides in [4] and agreement was confirmed. The AdS/CFT correspondence for orbifolds $\boldsymbol{S}^{5} / \Gamma$ was suggested in $[5,6]$. The agreement of the index for the orbifolds $\boldsymbol{S}^{5} / \mathbb{Z}_{n}$ with an $A_{n-1}$ type fixed locus $\boldsymbol{S}^{1} \subset \mathcal{Y}$ and the corresponding quiver gauge theories was confirmed in [7]. It was found that on the gravity side not only the gravity multiplet but also the tensor multiplets living on the fixed locus contribute to the index. The index of the Kaluza-Klein modes in $\mathrm{AdS}_{5} \times T^{1,1}$ was calculated in [8]. The large $N$ index of the corresponding quiver gauge theory, the Klebanov-Witten theory [9], was calculated in [10] and the agreement was confirmed.

If $N$ is finite the correspondence is modified for operators with dimension of order $N$ or larger. For example, in $\mathcal{N}=4 \mathrm{SO}(2 N)$ SYM the Pfaffian operators with dimension $N$ correspond not to Kaluza-Klein modes of supergravity fields but to D3-branes wrapped around topologically non-trivial cycles in $\boldsymbol{S}^{5} / \mathbb{Z}_{2}$ [11]. Similar relations hold for baryonic operators in quiver gauge theories whose dual geometries have topologically non-trivial three-cycles [12].

D3-branes play a role in finite $N$ corrections even when the internal space does not have topologically non-trivial three-cycles. In the case of $\mathcal{N}=4 \mathrm{U}(N) \mathrm{SYM}$ the one-toone correspondence between BPS operators and Kaluza-Klein modes is broken down for operators with dimension of order $N$ or larger. On the gauge theory side this is because single trace operators with the length $L>N$ are not independent but decomposable into shorter single-trace operators. We can explain this on the gravity side by assuming that the operators correspond not to point-like gravitons but to giant gravitons: D3-branes wrapped on topologically trivial three-cycles in $\boldsymbol{S}^{5}$. 1/2 BPS giant gravitons were constructed in [13]. Their angular momentum $J$ in $\boldsymbol{S}^{5}$ has the upper bound $J \leq N$, and the absence of giant gravitons with $J>N$ corresponds to the absence of independent single-trace operators with the length $L>N$. Indeed, the BPS partition function [14] of $\mathcal{N}=4 \mathrm{U}(N)$ SYM with finite $N$ was exactly reproduced in [15] based on the idea of [16] by the geometric quantization of $1 / 8$ BPS giant gravitons constructed in [17]. There is also a complementary way to reproduce the same BPS partition function [18] by using giant gravitons expanded in $\mathrm{AdS}_{5}[19,20]$. 
The result in [16] was extended in [21] to S-fold theories including the $\mathcal{N}=4 \mathrm{SO}(2 N)$ SYM. The exact BPS partition function was derived by the geometric quantization of BPS configurations of D3-branes in $\boldsymbol{S}^{5} / \mathbb{Z}_{k}$, where $\mathbb{Z}_{k}$ is the S-fold action transforming the $(p, q)$-string charges non-trivially. It is natural to attempt a similar derivation for the superconformal index, and indeed in [22] it was shown for the S-fold theories that the leading finite $N$ corrections can be reproduced as the index of fluctuation modes on D3branes wrapped around particular three-cycles. We mean in this paper by "the leading finite $N$ corrections" the corrections starting from $\mathcal{O}\left(q^{N}\right)$. In the case of S-fold theories we also have "the sub-leading corrections" starting from $\mathcal{O}\left(q^{2 N}\right)$, which was not studied in [22].

The purpose of this paper is to calculate the finite $N$ corrections to the superconformal index for ordinary orbifolds $\mathcal{Y}=\boldsymbol{S}^{5} / \widetilde{\Gamma}$, which have much more variety than the S-folds. (We use $\widetilde{\Gamma}$ for the orbifold group rather than $\Gamma$ and $\Gamma$ for the dual group $\operatorname{Hom}(\widetilde{\Gamma}, \mathrm{U}(1))$ because in the following we use the dual group more frequently than the orbifold group itself.) For an $\mathcal{N}=1$ supersymmetry to be preserved $\widetilde{\Gamma}$ must be a finite subgroup of $\mathrm{SU}(3)$ acting on $\mathbb{C}^{3}$ coordinates $(X, Y, Z) .{ }^{1}$ We restrict our attention to the toric case. Namely, we assume that $\widetilde{\Gamma}$ is abelian, and is a subset of the Cartan subgroup $H=\mathrm{U}(1)^{2} \subset \mathrm{SU}(3)$.

It is convenient to represent $h \in H$ as the $3 \times 3$ matrix acting on $(X, Y, Z)$ :

$$
w_{X}(h)^{R_{X}} w_{Y}(h)^{R_{Y}} w_{Z}(h)^{R_{Z}}=\left(\begin{array}{lll}
w_{X}(h) & & \\
& w_{Y}(h) & \\
& & w_{Z}(h)
\end{array}\right) .
$$

$R_{X}, R_{Y}$, and $R_{Z}$ are the Cartan generators of $s u(4) \sim$ so(6) acting on $X, Y$, and $Z$, respectively, and $w_{I}(h)(I=X, Y, Z)$ are complex numbers with the absolute value 1 depending on $h \in H$. For this to be an element of $\mathrm{SU}(3) w_{I}(h)$ must satisfy

$$
w_{X}(h) w_{Y}(h) w_{Z}(h)=1 \quad \forall h \in H .
$$

Generically the orbifolding breaks the $\mathcal{N}=4$ supersymmetry down to $\mathcal{N}=1$. Let $Q_{a}$ and $\bar{Q}^{\dot{a}}$ be the left-handed and the right-handed unbroken supercharges, respectively. To define the superconformal index we use $\bar{Q}^{\mathrm{i}}$ such that $\bar{\Delta}=2\left\{\left(\bar{Q}^{\mathrm{i}}\right)^{\dagger}, \bar{Q}^{\mathrm{i}}\right\}$ is given by

$$
\bar{\Delta}=2\left\{\left(\bar{Q}^{\mathrm{i}}\right)^{\dagger}, \bar{Q}^{\mathrm{i}}\right\}=H-2 \bar{J}-\left(R_{X}+R_{Y}+R_{Z}\right) .
$$

The index is defined by ${ }^{2}$

$$
\mathcal{I}\left(q, y, u_{I}, \zeta\right)=\operatorname{tr}\left[(-1)^{F} \bar{x}^{\bar{\Delta}} q^{H+\bar{J}} y^{2 J} \zeta^{\boldsymbol{b}} \prod_{I=X, Y, Z} u_{I}^{R_{I}}\right] .
$$

Only BPS operators saturating the BPS bound $\bar{\Delta} \geq 0$ contribute to the index and hence the index is independent of $\bar{x} . H$ is the dilatation and $J$ and $\bar{J}$ are the left- and rightangular momenta normalized so that the eigenvalues are quantized with unit $1 / 2 . u_{I}$ are

\footnotetext{
${ }^{1}$ We also use the notation $X_{I}$ which means $X, Y$, and $Z$ for $I=X, Y$, and $Z$.

${ }^{2}$ Our convention and notation for the fugacities are the same as those in [22] except for $y$, which is denoted in $[22]$ by $\widetilde{y}$.
} 
$\mathrm{SU}(3)$ fugacities satisfying $u_{X} u_{Y} u_{Z}=1$. We also use two independent variables $u$ and $v$ related to $u_{I}$ by $\left(u_{X}, u_{Y}, u_{Z}\right)=\left(u, \frac{v}{u}, \frac{1}{v}\right) . \boldsymbol{b}$ and $\zeta$ collectively represent baryonic charges and the corresponding fugacities, respectively, which will be discussed later in detail.

From the viewpoint on the gravity side this index is expected to be factorized into two factors:

$$
\mathcal{I}=\mathcal{I}^{\mathrm{KK}} \mathcal{I}^{\mathrm{D} 3}
$$

$\mathcal{I}^{\mathrm{KK}}$ is the contribution of Kaluza-Klein modes of massless fields, which has been already studied in the literature. $\mathcal{I}^{\mathrm{KK}}$ gives the exact index in the large $N$ limit. The purpose of this paper is to investigate the other factor, $\mathcal{I}^{\mathrm{D} 3}$, which gives finite $N$ corrections due to D3-branes wrapped on three-cycles in $\mathcal{Y}$. Again we focus only on the leading corrections starting from $\mathcal{O}\left(q^{N}\right)$, and do not pay attention to the sub-leading corrections starting from $\mathcal{O}\left(q^{k N}\right)$ with $k \geq 2$ depending on the sector we consider.

The rest of this paper is organized as follows. In the next section we summarize the toric diagrams and the quiver gauge theories for abelian orbifolds $\mathcal{Y}=\boldsymbol{S}^{5} / \widetilde{\Gamma}$. In section 3 we review how the superconformal index in the large $N$ limit is calculated on the gravity side as the contribution of Kaluza-Klein modes in the orbifold $\mathcal{Y}=\boldsymbol{S}^{5} / \widetilde{\Gamma}$. In section 4 we explain a prescription to obtain the leading finite $N$ corrections from wrapped D3-branes based on the analysis in [22]. We first discuss the relation between the wrapping number of D3-branes and the baryonic charges in the quiver gauge theory, and then we give a prescription to calculate the contribution in each wrapping sector. In section 5 we apply the method to examples with $N=2$ and confirm the agreement between the results of the localization and those of the D3-brane analysis. Section 6 is devoted to summary and discussions. The appendix contains results for $N=3$ for some of orbifolds in section 5 .

To write down indices we use characters associated with the spin and flavor symmetries. They are defined as follows.

The spin characters $\chi_{n}^{J}$ are defined by

$$
\chi_{n}^{J}=\frac{y^{n+1}-y^{-(n+1)}}{y-y^{-1}} .
$$

The $u(2)$ characters $\chi_{n}(a, b)$ are defined by

$$
\chi_{n}(a, b)=\frac{a^{n+1}-b^{n+1}}{a-b} .
$$

These are used to give the index of a theory with an $\mathrm{SU}(2)$ flavor symmetry. In all examples in this paper the $\mathrm{SU}(2)$ acts on $Y$ and $Z$, and the arguments of the characters are $u_{Y}=\frac{v}{u}$ and $u_{Z}=\frac{1}{v}$. We use the short-hand notation $\chi_{n}=\chi_{n}\left(\frac{v}{u}, \frac{1}{v}\right)$.

$\chi_{\left(r_{1}, r_{2}\right)}$ are the $s u(3)$ characters for representations with the Dynkin labels $\left(r_{1}, r_{2}\right)$. For the fundamental and the anti-fundamental representations these are given by

$$
\chi_{(1,0)}=\sum_{I=X, Y, Z} u_{I}, \quad \chi_{(0,1)}=\sum_{I=X, Y, Z} u_{I}^{-1}
$$




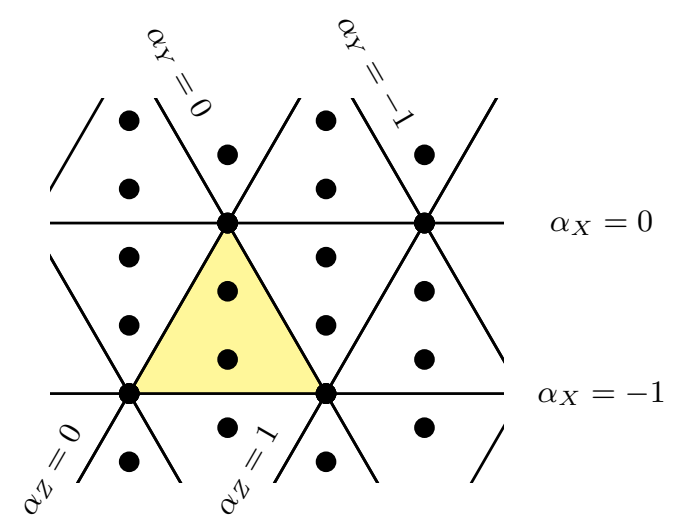

(a)

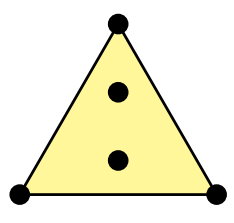

(b)

Figure 1. (a) The $\bar{H}$ plane with the lattice $P$ for the orbifold group $\widetilde{\Gamma}=\mathbb{Z}_{5}$ generated by $\operatorname{diag}\left(\omega_{5}^{-2}, \omega_{5}, \omega_{5}\right)$ is shown. $L$ is expressed as the set of intersections of three sets of parallel lines. The lattice $P$ is shown by dots. (b) A triangle picked up from (a) is shown. This is nothing but the toric diagram of the orbifold $\mathbb{C}^{3} / \mathbb{Z}_{5}$.

For a general representation $\left(r_{1}, r_{2}\right)$ it is given by

$$
\chi_{\left(r_{1}, r_{2}\right)}=\left|\begin{array}{ccc}
u_{X}^{r_{1}+1} & 1 & u_{X}^{-\left(r_{2}+1\right)} \\
u_{Y}^{r_{1}+1} & 1 & u_{Y}^{-\left(r_{2}+1\right)} \\
u_{Z}^{r_{1}+1} & 1 & u_{Z}^{-\left(r_{2}+1\right)}
\end{array}\right| /\left|\begin{array}{lll}
u_{X} & 1 & u_{X}^{-1} \\
u_{Y} & 1 & u_{Y}^{-1} \\
u_{Z} & 1 & u_{Z}^{-1}
\end{array}\right| .
$$

For later use we define $\omega_{n}$ by

$$
\omega_{n}=\exp \left(\frac{2 \pi i}{n}\right)
$$

\section{Orbifolds and quiver gauge theories}

\section{$2.1 \quad$ Toric diagrams}

Orbifolds we analyze in this paper are a special class of toric Calabi-Yaus, and their structure can be expressed by using toric diagrams. To define the toric diagram it is convenient to define $\alpha_{I}(I=X, Y, Z)$ by

$$
w_{I}=e^{2 \pi i \alpha_{I}}, \quad \alpha_{X}+\alpha_{Y}+\alpha_{Z}=0 .
$$

The parameters $\alpha_{I}$ can be regarded as (redundant) coordinates of the covering space $\bar{H}=\mathbb{R}^{2}$ of $H$. These are angular variables with the period 1 . Let $L$ be the associated lattice defined by $\alpha_{I} \in \mathbb{Z}$. $H$ and $\bar{H}$ are related by $H=\bar{H} / L$. In figure 1 (a) the lattice $L$ is expressed as the set of intersections of three sets of parallel lines $\alpha_{I} \in \mathbb{Z}$, which give a tessellation of the plane $\bar{H}$ by congruent triangles.

$\widetilde{\Gamma}$ is a finite subgroup of $H$, and is expressed as a lattice $P$ in $\bar{H}$, which includes $L$ as a sublattice. In other words, $P$ is a refinement of $L$. Thanks to the periodicity and the $\mathbb{Z}_{2}$ 
rotational symmetry of the lattice $P$ all triangles contain points in $P$ in the same way, and we can pick up one triangle to represent the orbifold group $\widetilde{\Gamma}$ (see (b) in figure 1). This is nothing but the toric diagram of the orbifold. ${ }^{3}$

Note that if we express $\widetilde{g} \in \widetilde{\Gamma}$ in the matrix form (1.1) each of diagonal components $w_{I}(\widetilde{g})$ is a one-dimensional representation of $\widetilde{\Gamma}$. Namely, $w_{I}$ can be regarded as elements of the dual group

$$
\Gamma=\operatorname{Hom}(\widetilde{\Gamma}, \mathrm{U}(1))
$$

Furthermore, the group $\Gamma$ is generated by the elements $w_{I}$. We can specify the orbifold $\mathcal{X}=\mathbb{C}^{3} / \widetilde{\Gamma}$ by giving a set of relations satisfied by $w_{I}$, which must always include $w_{X} w_{Y} w_{Z}=e$, where $e$ is the identity element of $\Gamma$.

\subsection{Quiver gauge theories}

The quiver gauge theory realized on an orbifold $\mathcal{X}=\mathbb{C}^{3} / \widetilde{\Gamma}$ is obtained by the standard prescription $[23,24]$ as follows. We start from $\mathcal{N}=4 \mathrm{SYM}$ with the gauge group $\mathrm{U}(|\Gamma| N)$, where $|\Gamma|$ is the order of $\Gamma$, which is the same as the order of $\widetilde{\Gamma}$. The $\mathcal{N}=4$ vector multiplet consists of an $\mathcal{N}=1$ vector multiplet $V$ and three $\mathcal{N}=1$ chiral multiplets $\Phi_{I}$ $(I=X, Y, Z)$. Because we consider abelian orbifolds we can discuss the projection on each $\mathcal{N}=1$ multiplet separately.

For the vector multiplet $V$ the action of $\widetilde{g} \in \widetilde{\Gamma}$ is

$$
\widetilde{g}: V \rightarrow V^{\prime}=u(\widetilde{g}) V u^{-1}(\widetilde{g}),
$$

where $u \in \operatorname{Hom}(\widetilde{\Gamma}, \mathrm{U}(|\Gamma| N))$ represents the action associated with a holonomy. In this paper we consider only the case that all gauge groups have the same rank, and it is realized by taking

$$
u(\widetilde{g})=R(\widetilde{g}) \otimes \mathbf{1}_{N},
$$

where $R(\widetilde{g})$ is the regular representation of $\widetilde{\Gamma}$. The regular representation of a finite group is the direct sum of all irreducible representations. Because $\widetilde{\Gamma}$ is abelian irreducible representations are identified with elements of the dual group (2.2) and the regular representation is given by

$$
R(\widetilde{g})=\bigoplus_{g \in \Gamma} g(\widetilde{g})
$$

Correspondingly, we divide $|\Gamma| N \times|\Gamma| N$ matrix $V$ into $|\Gamma|^{2}$ blocks $V_{g_{1} g_{2}}$ of size $N \times N$ labeled by $g_{1}, g_{2} \in \Gamma$. The action of $\widetilde{g} \in \widetilde{\Gamma}$ on each block is

$$
\widetilde{g}: V_{g_{1} g_{2}} \rightarrow V_{g_{1} g_{2}}^{\prime}=\frac{g_{1}(\widetilde{g})}{g_{2}(\widetilde{g})} V_{g_{1} g_{2}}
$$

\footnotetext{
${ }^{3}$ Usually a toric diagram is drawn so that $P$ is a square lattice. We do not do so and we draw the diagram as a regular triangle with dots.
} 
Then the projection leaves diagonal blocks $V_{g g}$. Let $\mathrm{U}(N)_{g}=\mathrm{SU}(N)_{g} \times \mathrm{U}(1)_{g}$ be the symmetry corresponding to the block $V_{g g}$. We define

$$
G=\prod_{g \in \Gamma} \mathrm{SU}(N)_{g}, \quad G_{B}^{0}=\prod_{g \in \Gamma} \mathrm{U}(1)_{g} .
$$

$G$ is the gauge group. The gauge fields for $G_{B}^{0}$ are decoupled in the IR and $G_{B}^{0}$ becomes global symmetry. In general $G_{B}^{0}$ is broken by anomalies to a subgroup $G_{B} \subset G_{B}^{0}$.

For the chiral multiplets the orbifold action is the composition of the $\mathbb{C}^{3}$ rotation given by (1.1) and the holonomy action like (2.3). Corresponding to the irreducible decomposition (2.5) the chiral multiplets are also decomposed into blocks $\left(\Phi_{I}\right)_{g_{1} g_{2}}$ of size $N \times N$. $\left(\Phi_{I}\right)_{g_{1} g_{2}}$ belongs to the bi-fundamental representation $(N, \bar{N})$ of $\mathrm{SU}(N)_{g_{1}} \times \mathrm{SU}(N)_{g_{2}}$, and in the following we use the notation $\Phi_{I}^{g_{1} \rightarrow g_{2}}$. The $\widetilde{g} \in \widetilde{\Gamma}$ action on each block is

$$
\widetilde{g}: \Phi_{I}^{g_{1} \rightarrow g_{2}} \rightarrow \Phi_{I}^{g_{1} \rightarrow g_{2}}=w_{I}(\widetilde{g}) \frac{g_{1}(\widetilde{g})}{g_{2}(\widetilde{g})} \Phi_{I}^{g_{1} \rightarrow g_{2}},
$$

and this block remains after the orbifold projection if and only if the relation

$$
w_{I} g_{1}=g_{2}
$$

holds. ${ }^{4}$ This condition determines the matter contents of the quiver gauge theory. Namely, we are left with $3|\Gamma|$ bi-fundamental fields $\Phi_{I}^{g \rightarrow w_{I} g}$ labeled by $I=X, Y, Z$ and $g \in \Gamma$. With this information it is easy to draw the quiver diagram. We first draw $|\Gamma|$ vertices corresponding to the elements of $\Gamma$. Each of them represents an $\mathrm{SU}(N)$ gauge group. A chiral multiplet belonging to the $(N, \bar{N})$ representation of $\mathrm{SU}(N)_{g_{1}} \times \mathrm{SU}(N)_{g_{2}}$ is represented as an arrow from the vertex $g_{1}$ to $g_{2}$. Because $\Gamma$ is a finite abelian group and can be given as a subgroup of $\mathrm{U}(1)^{2}$ it is natural and convenient to draw the diagram on the torus, and such a diagram is called a periodic quiver diagram.

Once we obtain the field contents of the quiver gauge theory we can in principle calculate the superconformal index for an arbitrary finite $N$ by the localization formula

$$
\mathcal{I}=\int d \mu \operatorname{Pexp}\left(\sum_{g \in \Gamma}\left(\mathbf{I}_{v}^{g}+\mathbf{I}_{X}^{g \rightarrow w_{X} g}+\mathbf{I}_{Y}^{g \rightarrow w_{Y} g}+\mathbf{I}_{Z}^{g \rightarrow w_{Z} g}\right)\right) .
$$

The plethystic exponential Pexp is defined as

$$
\operatorname{Pexp}\left(f\left(x_{i}\right)\right)=\exp \left(\sum_{n=1}^{\infty} \frac{1}{n} f\left(x_{i}^{n}\right)\right) .
$$

$\mathbf{I}_{v}^{g}$ is the single particle index of the $\mathrm{SU}(N)_{g}$ vector multiplet:

$$
\mathrm{I}_{v}^{g}=\left(-\frac{y q^{\frac{3}{2}}}{1-y q^{\frac{3}{2}}}-\frac{y^{-1} q^{\frac{3}{2}}}{1-y^{-1} q^{\frac{3}{2}}}\right) \chi_{\mathrm{adj}}^{g}
$$

\footnotetext{
${ }^{4}$ In this relation $w_{I}, g_{1}$, and $g_{2}$ are elements of $\Gamma$. Namely, this relation means that $w_{I}(\widetilde{g}) g_{1}(\widetilde{g})=g_{2}(\widetilde{g})$ holds for all $\widetilde{g} \in \widetilde{\Gamma}$.
} 
where $\chi_{\text {adj }}^{g}$ is the character of the adjoint representation of $\mathrm{SU}(N)_{g}$. The single-particle index of the chiral multiplet $\Phi_{I}^{g_{1} \rightarrow g_{2}}$ is

$$
\mathrm{I}_{I}^{g_{1} \rightarrow g_{2}}=\frac{q u_{I} \chi_{N}^{g_{1}} \chi_{N}^{g_{2}} \zeta_{g_{1}}^{\frac{1}{N}} \zeta_{g_{2}}^{-\frac{1}{N}}-q^{2} u_{I}^{-1} \chi_{N}^{g_{2}} \chi_{\bar{N}}^{g_{1}} \zeta_{g_{1}}^{-\frac{1}{N}} \zeta_{g_{2}}^{\frac{1}{N}}}{\left(1-y q^{\frac{3}{2}}\right)\left(1-y^{-1} q^{\frac{3}{2}}\right)}
$$

where $\chi_{N}^{g}$ and $\bar{\chi}_{N}^{g}$ are the characters of the fundamental and the anti-fundamental representations of $\mathrm{SU}(N)_{g}$. The explicit forms of the characters are

$$
\chi_{N}^{g}=\sum_{a=1}^{N} z_{a}^{g}, \quad \chi_{N}^{g}=\sum_{a=1}^{N} \frac{1}{z_{a}^{g}}, \quad \chi_{\mathrm{adj}}^{g}=\chi_{N}^{g} \chi_{N}^{g}-1 .
$$

Because the gauge groups are $\mathrm{SU}(N)$ the gauge fugacities $z_{a}^{g}(a=1, \ldots, N)$ are constrained by

$$
\prod_{a=1}^{N} z_{a}^{g}=1
$$

$\zeta_{g}$ are fugacities of $\mathrm{U}(1)_{g}$ baryonic symmetries. We denote the set of the $|\Gamma|$ fugacities collectively by $\zeta$ and it can be regarded as an element of the classical baryonic symmetry $G_{B}^{0}$. If the baryonic symmetry is anomalous and is broken to its subgroup, $\zeta$ takes only values in the anomaly-free subgroup.

$\int d \mu$ is the integration over the gauge fugacities $z_{a}^{g}$ defined by

$$
\int d \mu=\prod_{g \in \Gamma} \frac{1}{N !} \oint\left(\prod_{a=1}^{N-1} \frac{d z_{a}^{g}}{2 \pi i z_{a}^{g}}\right) \prod_{a \neq b}\left(1-\frac{z_{a}^{g}}{z_{b}^{g}}\right) .
$$

Although the single-particle indices (2.13) include fractional powers of $\zeta_{g}$ only integral powers remain after the integration over the gauge fugacities.

\subsection{Baryonic charges}

Let $\Lambda_{B}^{0}$ be the charge lattice associated with the classical baryonic symmetry $G_{B}^{0}$. An element of $\Lambda_{B}^{0}$ is specified by the set of $|\Gamma| \mathrm{U}(1)_{g}$ charges $B_{g} \in \mathbb{Z}$ satisfying

$$
\sum_{g \in \Gamma} B_{g}=0
$$

because all matter fields belong to bi-fundamental representations. ${ }^{5}$ Let $\boldsymbol{b}_{g}$ be the formal basis vector of the $\mathrm{U}(1)_{g}$ charge. A general element of $\Lambda_{B}^{0}$ is given by

$$
\boldsymbol{b}=\sum_{g \in \Gamma} B_{g} \boldsymbol{b}_{g}
$$

Due to $(2.17)$ the lattice $\Lambda_{B}^{0}$ is spanned by $|\Gamma|-1$ vectors $\boldsymbol{b}_{e}-\boldsymbol{b}_{g}(g \neq e)$.

\footnotetext{
${ }^{5}$ We normalize $B_{g}$ so that a field belonging to the $\mathrm{SU}(N)_{g}$ fundamental (anti-fundamental) representation carries $B_{g}=+1 / N(-1 / N)$.
} 
Now let us consider the effect of anomalies. If the theory is chiral, the presence of instantons causes violation of the conservation laws of the charges $B_{g}$. Let $N_{g}$ be the instanton numbers of $\mathrm{SU}(N)_{g}$. Then the baryonic charges change in this instanton background by

$$
\Delta B_{g}=\sum_{g^{\prime} \in \Gamma} n_{g \rightarrow g^{\prime}} N_{g^{\prime}}
$$

where $n_{g \rightarrow g^{\prime}}$ is the number of the arrows in the quiver diagram from $g$ to $g^{\prime}$. Arrows in the opposite direction are counted by -1 . A single $\mathrm{SU}(N)_{g}$ instanton changes the vector (2.18) by

$$
\Delta \boldsymbol{b}_{g} \equiv \sum_{g^{\prime}} \boldsymbol{b}_{g^{\prime}} n_{g^{\prime} \rightarrow g}
$$

For the orbifold quiver gauge theories this is given by

$$
\Delta \boldsymbol{b}_{g}=\sum_{I=X, Y, Z}\left(\boldsymbol{b}_{w_{I}^{-1} g}-\boldsymbol{b}_{w_{I} g}\right) .
$$

The quantum baryonic charge lattice $\Lambda_{B}$ taking account of this breaking of the conservation laws is given by

$$
\Lambda_{B}=\Lambda_{B}^{0} / \stackrel{A}{\sim}
$$

where $\stackrel{A}{\sim}$ is the equivalence relation defined by

$$
\Delta \boldsymbol{b}_{g} \stackrel{A}{\sim} 0 \quad \forall g \in \Gamma
$$

$\zeta^{\boldsymbol{b}}$ in the definition of the index (1.4) is a short-hand notation for $\prod_{g \in \Gamma} \zeta_{g}^{B_{g}}$. If we treat fugacities $\zeta_{g}$ as independent variables among them we can extract the index $\mathcal{I}_{\boldsymbol{b}}$ for each $\boldsymbol{b}$ by the expansion

$$
\mathcal{I}=\sum_{b \in \Lambda_{B}^{0}} \zeta^{b} \mathcal{I}_{\boldsymbol{b}}
$$

However, the physical meaning of $\mathcal{I}_{\boldsymbol{b}}$ is not clear because the classical baryonic charges are not conserved due to the anomalies. Instead of defining the index for each $\boldsymbol{b}$ we should treat equivalence classes $\widehat{\boldsymbol{b}} \in \Lambda_{B}$ as conserved charges and define the index for each of such classes by

$$
\mathcal{I}_{\widehat{b}}=\sum_{b \in \widehat{b}} \mathcal{I}_{\boldsymbol{b}}
$$

The index for a class $\widehat{\boldsymbol{b}}$ can be directly extracted from the index (1.4) by an expansion similar to (2.24). We impose the conditions

$$
\zeta^{\Delta \boldsymbol{b}_{g}}=1 \quad \forall g \in \Gamma
$$


on the fugacities $\zeta$. This is equivalent to the requirement that $\zeta$ is an element of the anomaly free baryonic symmetry group $G_{B} \subset G_{B}^{0}$. Then $\zeta^{\boldsymbol{b}}$ depends on $\boldsymbol{b}$ only through the equivalence class to which $\boldsymbol{b}$ belongs and we denote it by $\widehat{\zeta}$. We can rewrite the expansion (2.24) as

$$
\mathcal{I}=\sum_{\widehat{b} \in \Lambda_{B}} \zeta^{\widehat{b}} \mathcal{I}_{\widehat{\boldsymbol{b}}}
$$

\section{$3 \quad$ Large $N$ limit}

In this section we review known results for the superconformal index in the large $N$ limit. As is mentioned in the introduction it is given on the $\operatorname{AdS}$ side by $\mathcal{I}^{\mathrm{KK}}$, the contribution of Kaluza-Klein modes of massless fields. We can divide this into two factors $\mathcal{I}^{\text {grav }}$ and $\mathcal{I}^{\text {tensor }}$ : the index of the gravity multiplet in the bulk and the index of the tensor multiplets localized on the fixed loci.

\subsection{Gravity multiplet}

Let us first consider the gravity multiplet contribution $\mathcal{I}^{\text {grav }}$. It is given by

$$
\mathcal{I}^{\text {grav }}=\operatorname{Pexp}\left(\left.\mathcal{P}\right|^{\text {grav }}\right),
$$

where $\mathcal{P}$ is the orbifold projection operator and I $^{\text {grav }}$ is the single-particle index of the Kaluza-Klein modes in $\mathrm{AdS}_{5} \times \boldsymbol{S}^{5}$. For $\boldsymbol{S}^{5}$ without orbifolding the modes belong to the series of $\mathcal{N}=4$ superconformal multiplets $\mathcal{B}_{[0, n, 0](0,0)}^{\frac{1}{2}, \frac{1}{2}}(n=1,2, \ldots)[25,26]$. (See [27] for the notation for the superconformal representations.) The corresponding index is given by $[4]$

$$
\sum_{n=1}^{\infty} \mathrm{I}_{\mathcal{B}_{[0, n, 0](0,0)}^{\frac{1}{2}, \frac{1}{2}}}=\frac{u q}{1-u q}+\frac{\frac{v}{u} q}{1-\frac{v}{u} q}+\frac{\frac{1}{v} q}{1-\frac{1}{v} q}-\frac{y q^{\frac{3}{2}}}{1-y q^{\frac{3}{2}}}-\frac{\frac{1}{y} q^{\frac{3}{2}}}{1-\frac{1}{y} q^{\frac{3}{2}}} .
$$

The same index is obtained as the large $N$ limit of the index of $\mathcal{N}=4 \mathrm{SYM}$ with the gauge group $\mathrm{U}(N)$. Because we consider quiver gauge theories with the gauge group $\mathrm{SU}(N)^{|\Gamma|}$,

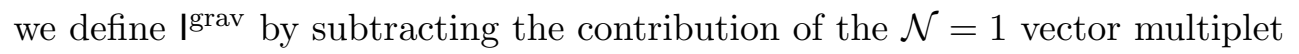

$$
\mathrm{I}_{\mathrm{vec}}=-\frac{y q^{\frac{3}{2}}}{1-y q^{\frac{3}{2}}}-\frac{\frac{1}{y} q^{\frac{3}{2}}}{1-\frac{1}{y} q^{\frac{3}{2}}}
$$

Namely, we define Irav $^{\text {by }}$

$$
\mathrm{I}^{\mathrm{grav}}=\frac{u q}{1-u q}+\frac{\frac{v}{u} q}{1-\frac{v}{u} q}+\frac{\frac{1}{v} q}{1-\frac{1}{v} q}=\sum_{I=1}^{3} \frac{u_{I} q}{1-u_{I} q} .
$$

We define the projection operator $\mathcal{P}$ so that it picks up the $\widetilde{\Gamma}$ invariant terms from the index. Let $f$ be a single-particle index defined by summing up modes in $\boldsymbol{S}^{5}$ :

$$
f\left(q, y, u_{I}\right)=\operatorname{tr}_{\boldsymbol{S}^{5}}\left(\cdots \prod_{I=X, Y, Z} u_{I}^{R_{I}}\right)
$$


We here focus on the dependence on the $\mathrm{SU}(3)$ fugacities $u_{I}$. Then the projected index $\mathcal{P} f$ is given by

$$
\begin{aligned}
\mathcal{P} f\left(q, y, u_{I}\right) & =\operatorname{tr}_{\boldsymbol{S}^{5}}\left(\frac{1}{|\Gamma|} \sum_{\widetilde{g} \in \widetilde{\Gamma}} \widetilde{g} \cdots \prod_{I=X, Y, Z} u_{I}^{R_{I}}\right) . \\
& =\frac{1}{|\Gamma|} \sum_{\widetilde{g} \in \widetilde{\Gamma}} \operatorname{tr}_{\boldsymbol{S}^{5}}\left(\cdots \prod_{I=X, Y, Z}\left(w_{I}(\widetilde{g}) u_{I}\right)^{R_{I}}\right) \\
& =\frac{1}{|\Gamma|} \sum_{\widetilde{g} \in \widetilde{\Gamma}} f\left(q, y, w_{I}(\widetilde{g}) u_{I}\right) .
\end{aligned}
$$

At the second equality we used $\widetilde{g}=\prod_{I=X, Y, Z} w_{I}(\widetilde{g})^{R_{I}}$.

\subsection{Tensor multiplet}

The tensor multiplet contribution $\mathcal{I}^{\text {tensor }}$ is present when the orbifold has fixed loci [7].

The presence of fixed loci and their types are easily read off from the toric diagram. The orbifold $\mathcal{Y}=\boldsymbol{S}^{5} / \widetilde{\Gamma}$ has fixed points if there exist $\widetilde{g} \in \widetilde{\Gamma}$ such that $\widetilde{g} \neq \widetilde{e}$ and $w_{I}(\widetilde{g})=1$ for one of $I=X, Y, Z$. In the toric diagram such an element is expressed as a dot on an open edge. (By an edge side we mean an edge with two endpoints excluded.) For example, if there are $k-1$ dots on an open edge and the edge is divided by them into $k$ pieces, then the $k-1$ elements of $\widetilde{\Gamma}$ associated with the dots together with the identity element form $\mathbb{Z}_{k} \subset \widetilde{\Gamma}$ acting only on two coordinates, and the fixed locus is the set of $A_{k-1}$ type singularities.

Let us consider the orbifold $\mathcal{X}=\mathbb{C} \times \mathbb{C}^{2} / \mathbb{Z}_{n}$ with the orbifold group generated by $\operatorname{diag}\left(1, \omega_{n}, \omega_{n}^{-1}\right)$. In this case an $\mathcal{N}=2$ supersymmetry is preserved. This has the $A_{n-1^{-}}$ type fixed locus $\boldsymbol{S}^{1} \subset \mathcal{Y}$ given by $Y=Z=0$. In the six-dimensional space $\operatorname{AdS}_{5} \times \boldsymbol{S}^{1}$ the $A_{n-1}$ type $\mathcal{N}=(2,0)$ theory lives, and $n-1$ tensor multiplets in the theory contribute to the index [7]. The Kaluza-Klein modes of a single tensor multiplet belong to the series of $\mathcal{N}=2$ superconformal representations $\mathcal{E}_{m(0,0)}(m=1,2,3, \ldots)$, and the corresponding single-particle index is

$$
\sum_{m=1}^{\infty} \boldsymbol{I}_{\mathcal{E}_{m(0,0)}}=\frac{u q}{1-u q}-\frac{y q^{\frac{3}{2}}}{1-y q^{\frac{3}{2}}}-\frac{\frac{1}{y} q^{\frac{3}{2}}}{1-\frac{1}{y} q^{\frac{3}{2}}} .
$$

(We can easily calculate this index by using the method in [28].) Similarly to the gravity multiplet contribution, we subtract the contribution of the $\mathcal{N}=1$ vector multiplet (3.3), and define $I_{X}^{\text {tensor }}$ by

$$
\mathrm{I}_{X}^{\mathrm{tensor}}=\frac{u q}{1-u q}
$$

In [7] this was extracted from the index of the gauge theory associated with the orbifold. We can generalize this to other fixed loci appearing along intersections of $\boldsymbol{S}^{5}$ and $X_{I}$ planes. 
We denote the corresponding single-particle index by $\left.\right|_{I} ^{\text {tensor }}$, and it is given by

$$
\mathrm{I}_{I}^{\mathrm{tensor}}=\frac{u_{I} q}{1-u_{I} q}
$$

Interestingly, these are the same as terms appearing in I $^{\text {grav }}$ in (3.4).

If $\mathbb{Z}_{n}$ is a proper subgroup of $\widetilde{\Gamma}$ and $\widetilde{\Gamma}$ includes elements that act non-trivially on the fixed locus, we need to perform the corresponding projection. It is realized by the projection $\mathcal{P}$ defined in (3.6). In a general situation in which the orbifold has type $A_{n_{I}-1}$ singular locus on the $X_{I}$-plane the total contribution of the tensor multiplets is given by

$$
\mathcal{I}^{\text {tensor }}=\prod_{I=X, Y, Z}\left(\mathcal{I}_{I}^{\text {tensor }}\right)^{n_{I}-1}, \quad \mathcal{I}_{I}^{\text {tensor }}=\operatorname{Pexp}\left(\mathcal{P I}_{I}^{\text {tensor }}\right) .
$$

\section{Finite $N$ corrections from wrapped D3-branes}

The purpose of this section is to give the prescription to calculate the leading finite $N$ corrections to the index from wrapped D3-branes based on some assumptions. The wrapping number of D3-branes in $\mathcal{Y}$ is related to the baryonic charges. We first define the wrapping number and then establish the relation to the baryonic charges.

\subsection{Wrapping number}

Let us define $\boldsymbol{S}^{5}$ as the subset of $\mathbb{C}^{3}$ by

$$
|X|^{2}+|Y|^{2}+|Z|^{2}=1
$$

and represent the worldvolume of a D3-brane in $\boldsymbol{S}^{5}$ as the intersection of a surface

$$
F(X, Y, Z)=0
$$

in $\mathbb{C}^{3}$ and the $\boldsymbol{S}^{5}$. For the D3-brane to be BPS the function $F$ must be holomorphic [17]. Here we are interested in topological aspects and the holomorphy is not assumed.

When we consider D3-branes in the orbifold $\mathcal{Y}=\boldsymbol{S}^{5} / \widetilde{\Gamma}$ the surface (4.2) must be invariant under the orbifold action. This requires the invariance of function $F$ up to an overall factor:

$$
F\left(w_{X}(\widetilde{g}) X, w_{Y}(\widetilde{g}) Y, w_{Z}(\widetilde{g}) Z\right)=w(\widetilde{g}) F(X, Y, Z) \quad \forall \widetilde{g} \in \widetilde{\Gamma},
$$

where $w_{I}(\widetilde{g})$ are diagonal elements in the matrix form (1.1) of $\widetilde{\Gamma}$ and $w(\widetilde{g})$ is a phase factor depending on $\widetilde{g}$. Note that $w$ as well as $w_{I}$ is an element of the dual group (2.2). A continuous deformation of the worldvolume is realized by changing the function $F$ continuously without violating the relation (4.3). Because $w$ takes discrete values, it does not change under continuous deformations. Namely, $w$ is a topological invariant associated with the D3-brane worldvolume in $\mathcal{Y}$. We call $w$ the wrapping number (although it is not an integer but an element of $\Gamma$ ). 
We will later focus on brane configurations described by monomial holomorphic functions $F\left(X_{I}\right)$. If $F\left(X_{I}\right)$ is such a function, by setting $X=Y=Z=1$ in (4.3) we obtain

$$
w=F\left(w_{X}, w_{Y}, w_{Z}\right)
$$

This relation directly gives the wrapping number of the configuration given by the function $F$. In particular, we obtain $w=w_{I}$ for $F=X_{I}$. Namely, the diagonal components in the orbifold action (1.1) are nothing but the wrapping numbers of three brane configurations $X_{I}=0$ which will play a central role in the following analysis.

If the orbifold has fixed loci there are shrinking two-cycles along the loci. The above definition of the wrapping number does not take these shrinking cycles into account. D3branes wrapped around these cycles are regarded as tensionless strings in the $(2,0)$ theories. The analysis in [7] showed that the index of the gauge theory in the large $N$ limit is reproduced as the contribution of the gravity multiplet in the bulk and the tensor multiplets localized on the loci, and the tensionless strings do not contribute the index.

To fully specify a D3-brane classical configuration we need to give not only the shape of the worldvolume but also the gauge field on the brane. Let us consider a D3-brane wrapped on $X_{I}=0$. The topology of the worldvolume is $\mathcal{C}=S^{3} / \widetilde{\Gamma}$, which may have fixed loci. The flat connection on $\mathcal{C}_{*}$ is classified by $H^{1}(\mathcal{C}, \mathrm{U}(1))=\Gamma{ }^{6}$

For general monomial functions

$$
F\left(X_{I}\right)=\prod_{I=X, Y, Z}\left(X_{I}\right)^{n_{I}}
$$

the equation $F\left(X_{I}\right)=0$ gives a set of three stacks of D3-branes. A stack wrapped on $X_{I}=0$ contains $n_{I} \mathrm{D} 3$-branes, and $\mathrm{U}\left(n_{I}\right)$ gauge theory is realized on it. We can specify a flat $\mathrm{U}(1)^{n_{I}} \subset \mathrm{U}\left(n_{I}\right)$ connection on each stack by a set of $n_{I}$ elements of $\Gamma$ :

$$
h_{I}=\left\{h_{I, 1}, h_{I, 2}, \ldots, h_{I, n_{I}}\right\}, \quad h_{I, i} \in \Gamma .
$$

These holonomy variables are shifted by the global 1-form symmetry associated with the background NS-NS two-form field, which we call $T$-symmetry. This symmetry is parameterized by $t \in H^{1}\left(\mathcal{Y}_{*}, \mathrm{U}(1)\right)=\Gamma,{ }^{7}$ and it acts on the holonomy variables as $h_{I, i} \rightarrow t h_{I, i}$.

\subsection{Wrapped D3-branes and baryonic charges}

Based on the results of the analysis of S-fold theories in [22] we assume that the finite $N$ corrections can be obtained from D3-branes wrapped around particular three-cycles $X=0$, $Y=0$, and $Z=0$.

The relation between wrapped D3-branes and baryonic operators was first pointed out in [11]. In general a wrapped D3-brane has non-trivial topology, and then we need to take

\footnotetext{
${ }^{6}$ If $\mathcal{C}$ contains an $A_{n+1}$ type singular locus it should be treated in this equation as a curve with mod $n$ linking. This is because if we move an endpoint of an open string attached on the worldvolume around the singular locus $n$ times the resulting open string can be continuously deformed back into the original configuration.

${ }^{7} \mathcal{Y}_{*}$ is defined from $\mathcal{Y}$ by removing fixed loci.
} 
account of the holonomy of the gauge field on the brane [12]. We can easily generalize the relation between wrapped branes and baryonic operators to a general orbifold.

Let us consider a D3-brane wrapped over one of the cycles $X_{I}=0$. The flat connection on the worldvolume is specified by one holonomy variable $h \in \Gamma$. Namely, the classical configuration is specified by $I$ and $h$. On the gauge theory side we have the corresponding baryonic operator ${ }^{8}$

$$
\mathcal{B}_{I, h}=\operatorname{det}\left(\Phi_{I}^{h \rightarrow w_{I} h}\right)
$$

This identification is consistent with the fact that on the gauge theory side we can identify the $T$-symmetry as the shift symmetry of the periodic quiver diagram. (We assume that the marginal deformation parameters such as gauge couplings are appropriately tuned.) An element $t \in \Gamma$ acts on the basis vectors of the baryonic charges as $\boldsymbol{b}_{g} \rightarrow \boldsymbol{b}_{t g}$.

As a simple check we can easily confirm that the dimension $\operatorname{dim} \mathcal{B}_{I, h}=N$ is reproduced as the mass of the D3-brane. For the orbifold $\mathcal{Y}=\boldsymbol{S}^{5} / \widetilde{\Gamma}$ the common radius $L$ of the $\boldsymbol{S}^{5}$ and the $\mathrm{AdS}_{5}$ is given in terms of the D3-brane tension $T_{\mathrm{D} 3}$ by

$$
L^{4}=\frac{|\Gamma| N}{2 \pi^{2} T_{\mathrm{D} 3}} .
$$

The mass of a D3-brane wrapped over one of the cycles $X_{I}=0$ is $M=2 \pi^{2} L^{3} T_{\mathrm{D} 3} /|\Gamma|=N / L$, and the dimension of the corresponding operator is $L M=N$. We can also show that the wrapped brane carries the correct R-charges $\left(R_{I}, R_{I+1}, R_{I+2}\right)=(N, 0,0),{ }^{9}$ due to the coupling to the background R-R flux [13].

We can generalize this correspondence to the configuration described by a general monomial function $F(X, Y, Z)=X^{n_{X}} Y^{n_{Y}} Z^{n_{Z}}$. On the gravity side $F(X, Y, Z)=0$ gives $n_{X}$ D3-branes wrapped on $X=0, n_{Y}$ D3-branes wrapped on $Y=0$, and $n_{Z}$ D3-branes wrapped on $Z=0$. Let $\vec{h}_{I}=\left\{h_{I, 1}, \ldots, h_{I, n}\right\}$ be the set of $n_{I}$ elements of $\Gamma$ representing the holonomy on the branes wrapped on $X_{I}=0$. A corresponding operator is

$$
\mathcal{O}=\prod_{I=X, Y, Z} \prod_{i=1}^{n_{I}} \mathcal{B}_{I, h_{I, i}} .
$$

By reading off the baryonic charges from (4.9) we obtain the map from the brane configuration with the holonomy on it to the baryonic charges:

$$
\boldsymbol{b}=\sum_{I=X, Y, Z} \sum_{i=1}^{n_{I}}\left(\boldsymbol{b}_{h_{I, i}}-\boldsymbol{b}_{w_{I} h_{I, i}}\right) .
$$

\footnotetext{
${ }^{8}$ Precisely, there is an ambiguity associated with the choice of origin of the holonomy variables, and (4.7) should be replaced with $\mathcal{B}_{I, h}=\operatorname{det}\left(\Phi_{I}^{c_{I} h \rightarrow c_{I} w_{I}{ }^{h}}\right)$, where $c_{I}$ are elements of $\Gamma$ depending on the choice of the origin. We cannot fix $c_{I}$ only by the consistency with the $T$-symmetry. For each $I$ the choice of $c_{I}$ is a matter of convention. However, relative values amomg $c_{I}$ are physical. In this paper we focus on configurations with a single wrapped brane and the corresponding indices are not affected by $c_{I}$. For this reason we simply neglect this ambiguity in the following.

${ }^{9}$ We treat $I=X, Y, Z$ as a cyclic variable. For example, if $I=Z,\left(R_{I}, R_{I+1}, R_{I+2}\right)=\left(R_{Z}, R_{X}, R_{Y}\right)$.
} 
Now let us establish the relation between the wrapping number $w \in \Gamma$ and the baryonic charge $\boldsymbol{b} \in \Lambda_{B}$. In general $\Lambda_{B}$ is larger than $\Gamma$ and we cannot simply identify $\boldsymbol{b}$ and $w$. This difference comes from the holonomy degrees of freedom on wrapped D3-branes. To relate the baryonic charges to the wrapping number we need to eliminate the information associated with the $T$-symmetry from the baryonic charges. Let us define the reduced lattice $\Lambda_{B}^{\text {red }}$ from $\Lambda_{B}$ by forgetting about the information associated with $T$ symmetry:

$$
\Lambda_{B}^{\mathrm{red}}=\Lambda_{B} / \stackrel{T}{\sim}
$$

where the equivalence relation $\stackrel{T}{\sim}$ is defined by

$$
\boldsymbol{b}_{g_{1}}-\boldsymbol{b}_{g_{2}} \stackrel{T}{\sim} \boldsymbol{b}_{t g_{1}}-\boldsymbol{b}_{t g_{2}} \quad t, g_{1}, g_{2} \in \Gamma
$$

In fact, we can easily show $\Delta \boldsymbol{b}_{g} \stackrel{T}{\sim} 0$ and hence

$$
\Lambda_{B}^{\mathrm{red}}=\Lambda_{B} / \stackrel{T}{\sim}=\Lambda_{B}^{0} / \stackrel{T}{\sim}
$$

Up to the $T$-symmetry the sum of two basis vectors of the classically conserved baryonic charge lattice $\Lambda_{B}^{0}$ is given by

$$
\left(\boldsymbol{b}_{e}-\boldsymbol{b}_{g_{1}}\right)+\left(\boldsymbol{b}_{e}-\boldsymbol{b}_{g_{2}}\right) \stackrel{T}{\sim}\left(\boldsymbol{b}_{e}-\boldsymbol{b}_{g_{1}}\right)+\left(\boldsymbol{b}_{g_{1}}-\boldsymbol{b}_{g_{1} g_{2}}\right)=\boldsymbol{b}_{e}-\boldsymbol{b}_{g_{1} g_{2}} .
$$

This relation implies that the map $\boldsymbol{b}_{e}-\boldsymbol{b}_{g} \rightarrow g$ is an isomorphism, and $\Lambda_{B}^{\text {red }} \cong \Gamma$. We can simply identify $\Lambda_{B}^{\text {red }}$ with the group of the wrapping number. The homomorphism $\varphi$ from $\Lambda_{B}^{0}$ to $\Lambda_{B}^{\text {red }}$ is given by

$$
\varphi: \boldsymbol{b}=\sum_{g \in \Gamma} B_{g} \boldsymbol{b}_{g} \rightarrow w=\prod_{g \in \Gamma} g^{-B_{g}} .
$$

The index for a specific $w$ is given by

$$
\mathcal{I}_{w}=\sum_{\boldsymbol{b}} \mathcal{I}_{\boldsymbol{b}}
$$

where the summation is taken over $\boldsymbol{b}$ satisfying $\varphi(\boldsymbol{b})=w$. Just like $\mathcal{I}_{\boldsymbol{b}}$ and $\mathcal{I}_{\widehat{\boldsymbol{b}}}$ we can extract $\mathcal{I}_{w}$ from the total index $\mathcal{I}$ by the $\zeta$-expansion. For this purpose we impose the constraint

$$
\frac{\zeta_{g_{1}}}{\zeta_{g_{2}}}=\frac{\zeta_{t g_{1}}}{\zeta_{t g_{2}}}, \quad \forall g_{1}, g_{2}, t \in \Gamma
$$

corresponding to (4.12). Then $\boldsymbol{\zeta}^{\boldsymbol{b}}$ depends on $\boldsymbol{b}$ through the corresponding wrapping number $w=\varphi(\boldsymbol{b})$ and we denote it by $\zeta^{w}$. We can rewrite (2.24) as

$$
\mathcal{I}=\sum_{w \in \Gamma} \zeta^{w} \mathcal{I}_{w}
$$

Because of (2.17) $\mathcal{I}$ is invariant under the overall phase rotation $\zeta_{g} \rightarrow e^{i \theta} \zeta_{g}$. With this redundancy we can set $\zeta_{e}=1$. Then the relation (4.17) means that the map $g \rightarrow \zeta_{g}$ is a homomorphism. Namely, we can regard the fugacities satisfying (4.17) as an element of the orbifold group $\widetilde{\Gamma}$. $\zeta^{w}$ in (4.18) is nothing but the pairing of $\zeta \in \widetilde{\Gamma}$ and $w \in \Gamma$. 


\subsection{Index from wrapped D3-branes}

In the next section we calculate indices on the both sides of the duality for each sector specified by the wrapping number $w \in \Gamma$ and compare them. For distinction, we denote the index on the gauge theory side by $\mathcal{I}_{w}^{\text {gauge }}$ and the index on the gravity side by $\mathcal{I}_{w}^{\text {AdS }}$.

On the gravity side the index $\mathcal{I}_{w}^{\text {AdS }}$ is given by

$$
\mathcal{I}_{w}^{\mathrm{AdS}}=\mathcal{I}^{\mathrm{KK}} \sum_{F} \sum_{h} \mathcal{I}_{F, h}^{\mathrm{D} 3}
$$

where $\mathcal{I}_{F, h}^{\mathrm{D} 3}$ is the index of D3-branes with the worldvolume $F=0$ and the holonomy $h$ on it. The summation is taken over monomials $F$ satisfying $F\left(w_{I}\right)=w$ and holonomies $h$ for each wrapped D3-brane configuration $F=0$. If $F$ is a monomial of order $k$ the index is $\mathcal{O}\left(q^{k N}\right)$. If $k=1$ the holonomy varianble is a single element of $\Gamma$, while if $k \geq 2 h$ is a set of $k$ elements of $\Gamma$.

Because we are interested in the leading finite $N$ corrections we focus on the four monomials $F=1, X, Y$, and $Z$, which give contribution to the sectors with $w=e, w_{X}$, $w_{Y}$, and $w_{Z}$, respectively. For $w \neq e, w_{I}$ the index $\mathcal{I}_{w}$ is of order $\mathcal{O}\left(q^{2 N}\right)$ or higher, and we will not pay attention to them.

Let us first consider the case that $e$ and $w_{I}$ are all different.

The leading contribution to $\mathcal{I}_{e}^{\text {AdS }}$ is given by $F=1$, which gives no wrapped D3-branes and the index includes only the Kaluza-Klein contributions

$$
\mathcal{I}_{e}^{\mathrm{AdS}}=\mathcal{I}^{\mathrm{KK}}+\mathcal{O}\left(q^{k N}\right)
$$

where $k$ is the lowest order of non-trivial monomial satisfying $F\left(w_{I}\right)=e$.

On the gauge theory side the baryonic charge $\boldsymbol{b}=0$ gives the leading contribution to $\mathcal{I}_{e}^{\text {gauge }}$

$$
\mathcal{I}_{e}^{\text {gauge }}=\mathcal{I}_{0}^{\text {gauge }}+\mathcal{O}\left(q^{k N}\right)
$$

where $k$ is the same as in (4.20). By comparing (4.20) and (4.21) we obtain

$$
\mathcal{I}_{0}^{\text {gauge }}=\mathcal{I}^{\mathrm{KK}}+\mathcal{O}\left(q^{k N}\right) .
$$

The leading contribution to $\mathcal{I}_{w_{X}}^{\text {AdS }}$ is given by $F=X$, which gives a single D3-brane wrapped over $X=0$. The index on the gravity side is given by

$$
\mathcal{I}_{w_{X}}^{\mathrm{AdS}}=\mathcal{I}^{\mathrm{KK}} \sum_{h} \mathcal{I}_{X, h}^{\mathrm{D} 3}+\mathcal{O}\left(q^{k N}\right)
$$

where $k$ is the lowest order of monomials $F \neq X$ satisfying $F\left(w_{I}\right)=w_{X}$. The worldvolume theory on the single D3-brane is a U(1) gauge theory and no fields couple to the holonomy. Therefore, the index $\mathcal{I}_{X, h}$ is independent of $h$ and we can replace the summation over $h$ by the factor $|\Gamma|$ :

$$
\mathcal{I}_{w_{X}}^{\mathrm{AdS}}=|\Gamma| \mathcal{I}^{\mathrm{KK}} \mathcal{I}_{X, 0}^{\mathrm{D} 3}+\mathcal{O}\left(q^{k N}\right) .
$$


On the gauge theory side the baryonic charges of sectors giving leading contribution are $\boldsymbol{b}_{g}-\boldsymbol{b}_{w_{X} g}$. Due to the $T$-symmetry the index $\mathcal{I}_{\boldsymbol{b}_{g}-\boldsymbol{b}_{w_{X}}}^{\text {gauge }}$ does not depend on $g$, and this degeneracy gives the factor $|\Gamma|$ :

$$
\mathcal{I}_{w}^{\text {gauge }}=|\Gamma| \mathcal{I}_{\boldsymbol{b}_{e}-\boldsymbol{b}_{w_{X}}}^{\text {gauge }}+\mathcal{O}\left(q^{k N}\right) .
$$

By comparing (4.24) and (4.25) we obtain

$$
|\Gamma| \mathcal{I}_{\boldsymbol{b}_{e}-\boldsymbol{b}_{w_{X}}}=|\Gamma| \mathcal{I}^{\mathrm{KK}} \mathcal{I}_{X, 0}^{\mathrm{D} 3}+\mathcal{O}\left(q^{k N}\right) .
$$

We also have similar relations for $w=w_{Y}$ and $w=w_{Z}$.

The index for a D3-brane wrapped on $X_{I}=0$ was calculated in [22] for S-fold theories, and the result can be used for orbifolds, too, by simply replace the S-fold projection operator used in [22] by the orbifold projection defined in (3.6). $\mathcal{I}_{X_{I}, 0}^{\mathrm{D} 3}$ are given by

$$
\mathcal{I}_{X_{I}, 0}^{\mathrm{D} 3}=q^{N} u_{I}^{N} \operatorname{Pexp}\left(\left.\mathcal{P}\right|_{I} ^{\mathrm{D} 3}\right),
$$

where the single-particle indices are the same as what are used in [22]:

$$
\mathbf{I}_{I}^{\mathrm{D} 3}=\frac{\frac{1}{u_{I} q}-\left(y+\frac{1}{y}\right) \frac{1}{u_{I}} q^{\frac{1}{2}}-\left(u_{I+1}+u_{I+2}\right) q+\left(y+\frac{1}{y}\right) q^{\frac{3}{2}}+2 \frac{1}{u_{I}} q^{2}-q^{3}}{\left(1-u_{I+1} q\right)\left(1-u_{I+2} q\right)} .
$$

(We set the $\mathrm{U}(1)_{A}$ fugacity $\eta$ introduced in [22] to realize the S-fold action to be 1.)

The relations in (4.22) and (4.26) hold only when $e, w_{X}, w_{Y}, w_{Z}$ are all different. If some of them coincide, the corresponding index $\mathcal{I}_{w}$ is given as the sum of their contributions.

Let us consider the case that one of $w_{I}$, say $w_{X}$, is $e$. This is the case for the orbifold $\mathbb{C} \times \mathbb{C}^{2} / \mathbb{Z}_{n}$ with $\mathbb{Z}_{n}$ acting on $Y$ and $Z$. In this case the two configurations given by $F=1$ and $F=X$ contribute to $\mathcal{I}_{e}$ :

$$
\mathcal{I}_{e}=\mathcal{I}^{\mathrm{KK}}\left(1+|\Gamma| \mathcal{I}_{X, 0}^{\mathrm{D} 3}+\cdots\right)
$$

$w_{X}=e$ means that the wrapping number of the configuration $X=0$ is trivial and we can unwrap the brane by a continuous deformation. Correspondingly, the single particle index includes a term proportional to $q^{-1}$, which we call the tachyonic term.

$$
\mathcal{P} \mathrm{I}_{X}^{\mathrm{D} 3}=\frac{1}{u q}+\cdots
$$

When we calculate the plethystic exponential such a tachyonic term is treated as follows.

$$
\operatorname{Pexp}\left(\frac{1}{u q}\right)=\frac{1}{1-\frac{1}{u q}}=-\frac{u q}{1-u q}=-u q \operatorname{Pexp}(u q)
$$

Although the physical meaning of this is not clear it was found in [22] that this gives the correct leading finite $N$ corrections for $\mathcal{N}=4 \mathrm{SYM}$, and we simply assume that this works for orbifolds, too. 
If $w=w_{Y}=w_{Z} \neq e$, the system has $\mathrm{SU}(2)$ flavor symmetry mixing $Y$ and $Z$. This is the case if all dots in the toric diagram except ones at corners are aligned on a median. The two configurations $Y=0$ and $Z=0$ contribute to the $w$ sector;

$$
\mathcal{I}_{w}=\mathcal{I}^{\mathrm{KK}}\left(|\Gamma| \mathcal{I}_{Y, 0}^{\mathrm{D} 3}+|\Gamma| \mathcal{I}_{Z, 0}^{\mathrm{D} 3}+\cdots\right)
$$

In this case, two configurations $Y=0$ and $Z=0$ belong to the same homology class, and we can continuously deform them to each other via the intermediate configurations given by

$$
b Y+c Z=0 .
$$

The coefficients $b$ and $c$ are the homogeneous coordinates of the configuration space $\mathbb{C} \boldsymbol{P}^{1}$. In this case each of the single particle indices $\left.\mathcal{P}\right|_{Y} ^{\mathrm{D} 3}$ and $\left.\mathcal{P}\right|_{Z} ^{\mathrm{D} 3}$ includes a term proportional to $q^{0}$. We call such terms "zero-mode terms."

$$
\left.\mathcal{P}\right|_{Y} ^{\mathrm{D} 3}=\frac{u}{v^{2}}+\cdots,\left.\quad \mathcal{P}\right|_{Z} ^{\mathrm{D} 3}=\frac{v^{2}}{u}+\cdots .
$$

The plethystic exponentials of these zero-mode terms give the fractional factors

$$
\begin{aligned}
& \mathcal{I}_{Y, 0}^{\mathrm{D} 3}=\left.q^{N} \frac{v^{N}}{u^{N}} \operatorname{Pexp} \mathcal{P}\right|_{Y} ^{\mathrm{D} 3}=\frac{(v / u)^{N}}{1-u / v^{2}}\left(q^{N}+\cdots\right), \\
& \mathcal{I}_{Z, 0}^{\mathrm{D} 3}=\left.q^{N} \frac{1}{v^{N}} \operatorname{Pexp} \mathcal{P}\right|_{Z} ^{\mathrm{D} 3}=\frac{1 / v^{N}}{1-v^{2} / u}\left(q^{N}+\cdots\right) .
\end{aligned}
$$

These are combined into the character of the $\mathrm{SU}(2)$ flavor symmetry:

$$
\begin{aligned}
\mathcal{I}_{Y, 0}^{\mathrm{D} 3}+\mathcal{I}_{Z, 0}^{\mathrm{D} 3} & =\left(\frac{v^{N}}{u^{N}}+\frac{v^{N-2}}{u^{N-1}}+\cdots+\frac{1}{v^{N}}\right) q^{N}+\cdots \\
& =\chi_{N}\left(\frac{v}{u}, \frac{1}{v}\right) q^{N}+\cdots,
\end{aligned}
$$

where $\chi_{N}$ is the $u(2)$ character defined in (1.7).

If $w=w_{X}=w_{Y}=w_{Z}$ are satisfied and the system has the flavor symmetry $\mathrm{SU}(3)$. This is the case for $\mathbb{C}^{3}$ and $\mathbb{C}^{3} / \mathbb{Z}_{3}$. For $\mathbb{C}^{3} / \mathbb{Z}_{3}$ the index of the $w$-sector is given as the sum of three contributions up to higher order corrections:

$$
\mathcal{I}_{w}=\mathcal{I}^{\mathrm{KK}}\left(|\Gamma| \mathcal{I}_{X, 0}^{\mathrm{D} 3}+|\Gamma| \mathcal{I}_{Y, 0}^{\mathrm{D} 3}+|\Gamma| \mathcal{I}_{Z, 0}^{\mathrm{D} 3}+\cdots\right) .
$$

(For $\mathbb{C}^{3}, w_{X}=w_{Y}=w_{Z}=e$ and we have additional 1" in the parentheses on the right hand side in (4.37).) In this case the three wrapped brane configurations are continuously deformed among them through the intermediate configurations

$$
a X+b Y+c Z=0 .
$$

$a, b$, and $c$ are homogeneous coordinates in the configuration space $\mathbb{C} \boldsymbol{P}^{2}$. Corresponding to the continuous deformation each of $I_{X_{I}}^{\mathrm{D} 3}$ has two zero-mode terms. The corresponding fractional factors give the $\mathrm{SU}(3)$ character when the three contributions are summed. 


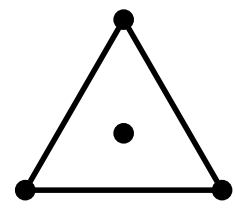

(a)

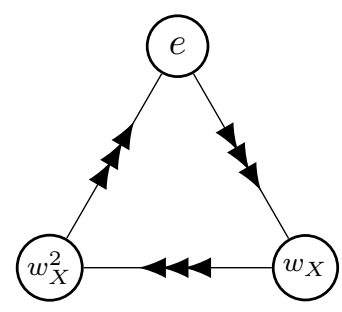

(b)

Figure 2. The toric diagram of $\mathbb{C}^{3} / \mathbb{Z}_{3}$ (a) and the corresponding quiver diagram (b) are shown.

\begin{tabular}{|ccc|}
\hline$w$ & $\mathcal{O}\left(q^{0}\right)$ and $\mathcal{O}\left(q^{N}\right)$ & higher order \\
\hline$e$ & 1 & $X^{3}, \ldots$ \\
$w_{X}$ & $X, Y, Z$ & $X^{4}, \ldots$ \\
$w_{X}^{2}$ & & $X^{2}, \ldots$ \\
\hline
\end{tabular}

Table 1. Wrapping numbers $w \in \Gamma$ and the corresponding monomials $F\left(X_{I}\right)$ are shown for the orbifold $\mathcal{X}=\mathbb{C}^{3} / \mathbb{Z}_{3}$. The shaded row is irrelevant to the leading corrections.

\section{$5 \quad$ Examples}

In this section we confirm that by the prescription given in the previous section we can obtain the leading finite $N$ corrections for $N=2$. The results for $N=3$ are shown in appendix A.

\section{$5.1 \mathbb{C}^{3} / \mathbb{Z}_{2 n+1}(n=1,2,3, \ldots)$}

Let us first consider the series of orbifolds $\mathcal{X}=\mathbb{C}^{3} / \mathbb{Z}_{2 n+1}(n=1,2,3, \ldots)$ with $\mathbb{Z}_{2 n+1}$ generated by $\operatorname{diag}\left(\omega_{2 n+1}^{-2}, \omega_{2 n+1}, \omega_{2 n+1}\right)$. The dual group $\Gamma=\mathbb{Z}_{2 n+1}$ is characterized by the relations

$$
w_{Y}=w_{Z}, \quad w_{X}=w_{Y}^{-2}, \quad w_{Y}^{2 n+1}=e .
$$

The $n=1$ case is special in the sense that $w_{X}$ agrees with $w_{Y}=w_{Z}$ then and the flavor symmetry is enhanced to $\mathrm{SU}(3)$. We give the results for $n=1,2$, and 3 .

\subsection{1 $\mathbb{C}^{3} / \mathbb{Z}_{3}$}

If $n=1$ the relations among $w_{I}$ are

$$
w_{X}=w_{Y}=w_{Z}, \quad w_{X}^{3}=e .
$$

The toric diagram and the quiver diagram are shown in figure 2. There are three sectors labeled by $w=e, w_{X}, w_{X}^{2}$ (see table 1 ).

Let us first consider the sector with trivial wrapping $w=e$, which we call the mesonic sector. The relations (5.2) show that any monomial $w_{X}^{n_{1}} w_{Y}^{n_{2}} w_{Z}^{n_{3}}$ of order $n_{1}+n_{2}+n_{3}=3 k$ $(k=0,1,2, \ldots)$ is $e$, and the corresponding function $F(X, Y, Z)=X^{n_{1}} Y^{n_{2}} Z^{n_{3}}$ gives a brane 
configuration $F=0$ contributing to the index of the mesonic sector. Such a monomial with the lowest order is $F=1$. The corresponding D3-brane configuration, $F=0$, does not contain any D3-brane, and the corresponding index is $\mathcal{I}_{1}^{\mathrm{D} 3}=1$. We show this monomial in the second column in table 1. There exist infinite number of higher order monomials which give higher order contributions to the index. An example of such higher order monomials with the lowest order, $X^{3}$, is shown in the third column in table 1 . This higher order contribution is $\mathcal{O}\left(q^{3 N}\right)$, and hence we have the relation $\mathcal{I}_{e}^{\mathrm{D} 3}=1+\mathcal{O}\left(q^{3 N}\right)$. On the gauge theory side, the operator corresponding to $F=1$ is the identity operator with the baryonic charge $\boldsymbol{b}=0$. For the higher order monomial like $F=X^{3}$, there are many operators carrying different baryonic charges depending on the holonomy. Because such higher order operators give indices of order $\mathcal{O}\left(q^{3 N}\right)$ or higher, we obtain the relation $\mathcal{I}_{e}^{\text {gauge }}=\mathcal{I}_{0}^{\text {gauge }}+\mathcal{O}\left(q^{3 N}\right)$. Combining these relations we obtain the relation

$$
\mathcal{I}_{0}^{\text {gauge }}=\mathcal{I}^{\text {grav }}+\mathcal{O}\left(q^{3 N}\right),
$$

which we want to check. We calculate the $\mathcal{I}_{0}^{\text {gauge }}$ on the left hand side for small $N$ by the localization formula. The result for $N=2$ is

$$
\begin{aligned}
\mathcal{I}_{0}^{\text {gauge }}= & 1+\left(1-\chi_{(1,1)}+\chi_{(3,0)}\right) q^{3} \\
& +\left(-2+\chi_{(0,3)}-\chi_{(1,1)}-\chi_{(3,0)}-2 \chi_{(4,1)}+2 \chi_{(6,0)}\right) q^{6}+\mathcal{O}\left(q^{\frac{15}{2}}\right) .
\end{aligned}
$$

$\mathcal{I}^{\text {grav }}$ on the right hand side in (5.3) is obtained by the formula (3.1). It is independent of $N$ and given by

$$
\begin{aligned}
\mathcal{I}^{\text {grav }}= & 1+\left(1-\chi_{(1,1)}+\chi_{(3,0)}\right) q^{3} \\
& +\left(1+\chi_{(0,3)}-\chi_{(1,1)}+2 \chi_{(3,0)}-2 \chi_{(4,1)}+2 \chi_{(6,0)}\right) q^{6}+\mathcal{O}\left(q^{9}\right)
\end{aligned}
$$

The comparison of (5.4) and (5.5) shows that the relation (5.3) certainly holds for $N=2$.

Next, let us consider the sectors with $w \neq e$. We call such sectors baryonic sectors. In the example of $\mathbb{C}^{3} / \mathbb{Z}_{3}$ we have two baryonic sectors with $w=w_{X}$ and $w=w_{X}^{2}$. For the former the corresponding functions $F$ with the lowest order are $F=X, F=Y$, and $F=Z$, which are shown in the second column in table 1. For each of D3-brane configurations $X=0, Y=0$, and $Z=0$, the holonomy on the brane is specified by an element of $\Gamma=\mathbb{Z}_{3}$. We should sum up contributions of three D3-brane configurations with all possible holonomies. They gives the index of order $\mathcal{O}\left(q^{N}\right)$. We also have higher order functions $F$. Among such functions an example with the lowest order, $X^{4}$, is shown in the third column in table 1. The D3-branes corresponding to such higher order functions give $\mathcal{O}\left(q^{4 N}\right)$ contributions to the index. Therefore, the D3-brane contribution for the $e=w_{X}$ sector is $\mathcal{I}_{w_{X}}^{\mathrm{D} 3}=3 \mathcal{I}_{X, 0}^{\mathrm{D} 3}+3 \mathcal{I}_{Y, 0}^{\mathrm{D} 3}+3 \mathcal{I}_{Z, 0}^{\mathrm{D} 3}+\mathcal{O}\left(q^{4 N}\right)$. On the gauge theory side, the operators corresponding to the brane configuration $X_{I}=0$ are $\operatorname{det}\left(\Phi_{I}^{g \rightarrow w_{X} g}\right)\left(g \in \mathbb{Z}_{3}\right)$, which have the baryonic charges $\boldsymbol{b}=\boldsymbol{b}_{g}-\boldsymbol{b}_{w_{X} g}$. Three sectors labeled by these baryonic charges give the same index due to the $T$-symmetry, and we obtain the relation $\mathcal{I}_{w_{X}}^{\text {gauge }}=$ $3 \mathcal{I}_{\boldsymbol{b}_{e}-\boldsymbol{b}_{w_{X}}}+\mathcal{O}\left(q^{4 N}\right)$. The discrepancy of order $\mathcal{O}\left(q^{4 N}\right)$ comes from the existence of other 


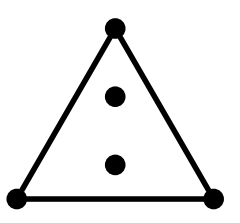

(a)

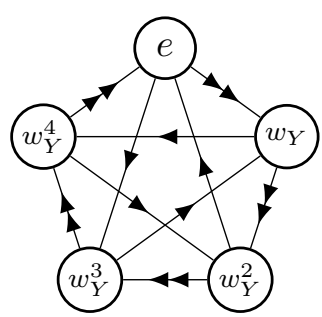

(b)

Figure 3. The toric diagram of $\mathbb{C}^{3} / \mathbb{Z}_{5}$ (a) and the corresponding quiver diagram (b) are shown.

baryonic charges corresponding to $w=w_{X}$. By combining the relations above we obtain

$$
\frac{\mathcal{I}_{\boldsymbol{b}_{e}-\boldsymbol{b}_{w_{X}}}^{\text {gauge }}}{\mathcal{I}_{0}^{\text {gauge }}}=\mathcal{I}_{X, 0}^{\mathrm{D} 3}+\mathcal{I}_{Y, 0}^{\mathrm{D} 3}+\mathcal{I}_{Z, 0}^{\mathrm{D} 3}+\mathcal{O}\left(q^{4 N}\right) .
$$

Let us confirm this relation by the numerical calculation for $N=2$. The result of the localization for the left hand side of (5.6) is

$$
\begin{aligned}
\frac{\mathcal{I}_{\boldsymbol{b}_{e}-\boldsymbol{b}_{w_{X}}}^{\text {gauge }}}{\mathcal{I}_{0}^{\text {gauge }}}= & \chi_{(2,0)} q^{2}+\chi_{1}^{J}\left(\chi_{(2,0)}-\chi_{(0,1)}\right) q^{\frac{7}{2}} \\
& +\left(\chi_{2}^{J}\left(\chi_{(2,0)}-\chi_{(0,1)}\right)-\left(\chi_{(0,1)}+\chi_{(1,2)}+\chi_{(2,0)}\right)\right) q^{5} \\
& +\left(\chi_{3}^{J}\left(\chi_{(2,0)}-\chi_{(0,1)}\right)+\chi_{1}^{J}\left(\chi_{(3,1)}-\chi_{(1,2)}\right)\right) q^{\frac{13}{2}} \\
& +\left(4 \chi_{(1,2)}+3 \chi_{(2,0)}+\chi_{4}^{J} \chi_{(2,0)}+\chi_{(2,3)}+3 \chi_{(3,1)}+\chi_{(5,0)}-\chi_{(6,1)}\right. \\
& \left.+\left(7+\chi_{2}^{J}-\chi_{4}^{J}\right) \chi_{(0,1)}\right) q^{8}+\mathcal{O}\left(q^{\frac{19}{2}}\right) .
\end{aligned}
$$

The right hand side of (5.6) is

$$
\begin{aligned}
\mathcal{I}_{X, 0}^{\mathrm{D} 3}+\mathcal{I}_{Y, 0}^{\mathrm{D} 3}+\mathcal{I}_{Z, 0}^{\mathrm{D} 3}= & \cdots(\text { identical terms }) \cdots \\
& +\left(\chi_{(1,2)}+\chi_{(2,0)}+\chi_{4}^{J} \chi_{(2,0)}+\chi_{(2,3)}+\chi_{(3,1)}-\chi_{(5,0)}-\chi_{(6,1)}\right. \\
& \left.+\left(4+\chi_{2}^{J}-\chi_{4}^{J}\right) \chi_{(0,1)}\right) q^{8}+\mathcal{O}\left(q^{\frac{19}{2}}\right) .
\end{aligned}
$$

In this equation "... (identical terms) ..." mean that the terms are the same as the corresponding terms in (5.7). Comparison of (5.7) and (5.8) confirm the relation (5.6).

For the other baryonic sector with the wrapping number $e=w_{X}^{2}$ the index is $\mathcal{O}\left(q^{2 N}\right)$. We do not analyze such sectors in this paper.

\subsection{2 $\mathbb{C}^{3} / \mathbb{Z}_{5}$}

Let us move on to the case with $n=2: \mathcal{X}=\mathbb{C}^{3} / \mathbb{Z}_{5}$. In this case $w_{X} \neq w_{Y}=w_{Z}$ and the system has a flavor symmetry $\mathrm{SU}(2)$. The toric diagram and the quiver diagram are shown in figure 3. There are five sectors labeled by the wrapping numbers $w=w_{Y}^{k}$ $(k=0,1,2,3,4)$. The order of indices of these five sectors can be easily read off from table 2. The sector with $w=e$ is the mesonic sector and sectors with $w=w_{Y}$ and $w=w_{Y}^{3}=w_{X}$ are baryonic sectors whose indices are $\mathcal{O}\left(q^{N}\right)$. The other sectors with 


\begin{tabular}{|ccc|}
\hline$w$ & $\mathcal{O}\left(q^{0}\right)$ and $\mathcal{O}\left(q^{N}\right)$ & higher order \\
\hline$e$ & 1 & $X Y^{2}, \ldots$ \\
$w_{Y}$ & $Y, Z$ & $X^{2}, \ldots$ \\
$w_{Y}^{2}$ & & $Y^{2}, \ldots$ \\
$w_{Y}^{3}$ & $X$ & $Y^{3}, \ldots$ \\
$w_{Y}^{4}$ & & $X Y, \ldots$ \\
\hline
\end{tabular}

Table 2. Wrapping numbers $w \in \Gamma$ and the corresponding monomials $F\left(X_{I}\right)$ are shown for the orbifold $\mathcal{X}=\mathbb{C}^{3} / \mathbb{Z}_{5}$. The shaded rows are irrelevant to the leading corrections.

$w=w_{Y}^{2}$ and $w=w_{Y}^{4}$, which are shaded in table 2 , give indices of $\mathcal{O}\left(q^{2 N}\right)$ and we do not discuss them.

The expected relations are

$$
\begin{aligned}
\mathcal{I}_{0}^{\text {gauge }} & =\mathcal{I}^{\text {grav }}+\mathcal{O}\left(q^{3 N}\right), \\
\frac{\mathcal{I}_{\boldsymbol{b}_{e}-\boldsymbol{b}_{w_{Y}}}^{\text {gauge }}}{\mathcal{I}_{0}^{\text {gauge }}} & =\mathcal{I}_{Y, 0}^{\mathrm{D} 3}+\mathcal{I}_{Z, 0}^{\mathrm{D} 3}+\mathcal{O}\left(q^{2 N}\right), \\
\mathcal{I}_{\boldsymbol{b}_{e}-\boldsymbol{b}_{w_{Y}^{3}}}^{\text {gauge }} & =\mathcal{I}_{X, 0}^{\mathrm{D} 3}+\mathcal{O}\left(q^{3 N}\right) . \\
\mathcal{I}_{0}^{\text {gauge }} &
\end{aligned}
$$

Let us confirm these relations for $N=2$. For the mesonic sector with $w=e$ the result of localization on the gauge theory side is

$$
\mathcal{I}_{0}^{\text {gauge }}=1+\left(u^{5}-\frac{\chi_{3}}{u}+\chi_{5}\right) q^{5}-5\left(u \chi_{2}+1\right) q^{6}+\mathcal{O}\left(q^{7}\right) .
$$

The formula for the Kaluza-Klein modes (3.1) gives

$$
\mathcal{I}^{\text {grav }}=\cdots(\text { identical terms }) \cdots+0 q^{6}+\mathcal{O}\left(q^{7}\right) .
$$

(5.10) and (5.11) are consistent with the first relation in (5.9).

For the baryonic sector with $w=w_{Y}$ the result of localization with $N=2$ is

$$
\begin{aligned}
\frac{\mathcal{I}_{\boldsymbol{b}_{e}-\boldsymbol{b}_{w_{Y}}}^{\text {gauge }}}{\mathcal{I}_{0}^{\text {gauge }}}= & \chi_{2} q^{2}+u^{2} \chi_{1} q^{3}+\chi_{1}^{J}\left(\chi_{2}-\frac{1}{u}\right) q^{\frac{7}{2}}+u^{4} q^{4} \\
& +\left(\chi_{2}^{J}\left(\chi_{2}-\frac{1}{u}\right)-\frac{3}{u}-\chi_{2}\right) q^{5}+2 \chi_{1}^{J} u^{4} q^{\frac{11}{2}}+\mathcal{O}\left(q^{6}\right),
\end{aligned}
$$

and on the gravity side we have

$$
\mathcal{I}_{Y, 0}^{\mathrm{D} 3}+\mathcal{I}_{Z, 0}^{\mathrm{D} 3}=\cdots(\text { identical terms }) \cdots+\chi_{1}^{J} u^{4} q^{\frac{11}{2}}+\mathcal{O}\left(q^{6}\right) .
$$

(5.12) and (5.13) satisfy the second relation in (5.9).

For the baryonic sector with $w=w_{Y}^{3}$ the result of localization with $N=2$ is

$$
\begin{aligned}
\frac{\mathcal{I}_{\boldsymbol{b}_{e}-\boldsymbol{b}_{w_{Y}^{3}}}^{\text {gauge }}}{\mathcal{I}_{0}^{\text {gauge }}}= & u^{2} q^{2}+u^{2} \chi_{1}^{J} q^{\frac{7}{2}}+u \chi_{3} q^{4}+u^{2}\left(\chi_{2}^{J}-1\right) q^{5}+\left(\frac{\chi_{2}}{u^{2}}+\chi_{6}\right) q^{6} \\
& +\left(u^{2} \chi_{3}^{J}-u^{2} \chi_{1}^{J}\right) q^{\frac{13}{2}}-\left(u^{2} \chi_{5}+4 u \chi_{3}+2 \chi_{1}\right) q^{7}+\mathcal{O}\left(q^{\frac{15}{2}}\right),
\end{aligned}
$$




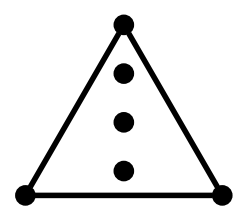

(a)

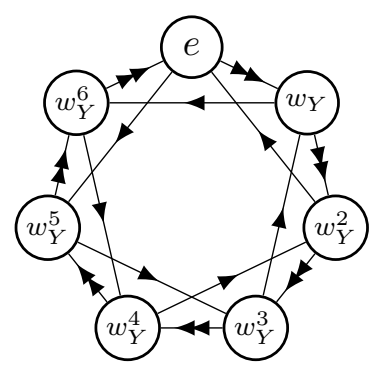

(b)

Figure 4. The toric diagram of $\mathbb{C}^{3} / \mathbb{Z}_{7}$ (a) and the corresponding quiver diagram (b) are shown.

\begin{tabular}{|ccc|}
\hline$w$ & $\mathcal{O}\left(q^{0}\right)$ and $\mathcal{O}\left(q^{N}\right)$ & higher order \\
\hline$e$ & 1 & $X Y^{2}, \ldots$ \\
$w_{Y}$ & $Y, Z$ & $X^{3}, \ldots$ \\
$w_{Y}^{2}$ & & $Y^{2}, \ldots$ \\
$w_{Y}^{3}$ & & $X^{2}, \ldots$ \\
$w_{Y}^{4}$ & & $X^{2} Y, \ldots$ \\
$w_{Y}^{5}$ & $X$ & $X^{2} Y^{2}, \ldots$ \\
$w_{Y}^{6}$ & & $X Y, \ldots$ \\
\hline
\end{tabular}

Table 3. Wrapping numbers $w \in \Gamma$ and the corresponding monomials $F\left(X_{I}\right)$ are shown for the orbifold $\mathcal{X}=\mathbb{C}^{3} / \mathbb{Z}_{7}$. The shaded rows are irrelevant to the leading corrections.

and on the gravity side we have

$$
\mathcal{I}_{X, 0}^{\mathrm{D} 3}=\cdots(\text { identical terms }) \cdots-\left(u^{2} \chi_{5}+u \chi_{3}\right) q^{7}+\mathcal{O}\left(q^{\frac{15}{2}}\right) .
$$

(5.14) and (5.15) satisfy the last relation in (5.9).

\subsection{3 $\mathbb{C}^{3} / \mathbb{Z}_{\mathbf{7}}$}

For $n=3$ the orbifold is $\mathcal{X}=\mathbb{C}^{3} / \mathbb{Z}_{7}$ with the orbifold group generated by $\operatorname{diag}\left(\omega_{7}^{-2}, \omega_{7}, \omega_{7}\right)$. The toric diagram and the quiver diagram are shown in figure 4 . There are seven sectors specified by the wrapping numbers $w=w_{Y}^{k}(k=0,1,2, \ldots, 6)$ (see table 3 ).

We are interested in the mesonic sector with $w=e$ and two baryonic sectors with $w=w_{Y}$ and $w=w_{Y}^{5}$. The other sectors shaded in table 3 give higher order corrections which we do not discuss here. The expected relations are

$$
\begin{aligned}
\mathcal{I}_{0}^{\text {gauge }} & =\mathcal{I}^{\text {grav }}+\mathcal{O}\left(q^{3 N}\right), \\
\frac{\mathcal{I}_{\boldsymbol{b}_{e}-\boldsymbol{b}_{w_{Y}}}^{\text {gauge }}}{\mathcal{I}_{0}^{\text {gauge }}} & =\mathcal{I}_{Y, 0}^{\mathrm{D} 3}+\mathcal{I}_{Z, 0}^{\mathrm{D} 3}+\mathcal{O}\left(q^{3 N}\right), \\
\frac{\mathcal{I}_{\boldsymbol{b}_{e}-\boldsymbol{b}_{w_{Y}^{5}}}^{\text {gauge }}}{\mathcal{I}_{0}^{\text {gauge }}} & =\mathcal{I}_{X, 0}^{\mathrm{D} 3}+\mathcal{O}\left(q^{4 N}\right) .
\end{aligned}
$$

Let us confirm these relations for $N=2$. 
For the mesonic sector with $w=e$ we have on the gauge theory side

$$
\mathcal{I}_{0}^{\text {gauge }}=1-7\left(u \chi_{2}+1\right) q^{6}+\mathcal{O}\left(q^{7}\right)
$$

and on the gravity side

$$
\mathcal{I}^{\text {grav }}=1+0 q^{6}+\mathcal{O}\left(q^{7}\right)
$$

These are consistent with the first relation in (5.16). For the baryonic sector with $w=w_{Y}$ the index on the gauge theory side is

$$
\begin{aligned}
\frac{\mathcal{I}_{\boldsymbol{b}_{e}-\boldsymbol{b}_{w_{Y}}}^{\text {gauge }}}{\mathcal{I}_{0}^{\text {gauge }}}= & \chi_{2} q^{2}+\chi_{1}^{J}\left(\chi_{2}-\frac{1}{u}\right) q^{\frac{7}{2}}+u^{3} \chi_{1} q^{4}+\left(\chi_{2}^{J} \chi_{2}-\frac{\chi_{2}^{J}}{u}-\chi_{2}-\frac{3}{u}\right) q^{5} \\
& +u^{6} q^{6}+\chi_{3}^{J}\left(-\frac{1}{u}+\chi_{2}\right) q^{\frac{13}{2}}-6 u^{3} \chi_{1} q^{7}+\mathcal{O}\left(q^{\frac{15}{2}}\right)
\end{aligned}
$$

and on the gravity side we have

$$
\mathcal{I}_{Y, 0}^{\mathrm{D} 3}+\mathcal{I}_{Z, 0}^{\mathrm{D} 3}=\cdots(\text { identical terms }) \cdots-4 u^{3} \chi_{1} q^{7}+\mathcal{O}\left(q^{\frac{15}{2}}\right) .
$$

These are consistent with the second equation in (5.16). For the baryonic sector with $w=w_{Y}^{5}$ on the gauge theory side we have

$$
\begin{aligned}
\frac{\mathcal{I}_{\boldsymbol{b}_{e}-\boldsymbol{b}_{w_{Y}^{5}}}^{\text {gauge }}}{\mathcal{I}_{0}^{\text {gauge }}}= & u^{2} q^{2}+u^{2} \chi_{1}^{J} q^{\frac{7}{2}}+\left(u^{2} \chi_{2}^{J}-u^{2}\right) q^{5}+u \chi_{5} q^{6}+u^{2}\left(-\chi_{1}^{J}+\chi_{3}^{J}\right) q^{\frac{13}{2}} \\
& +u^{2}\left(1-\chi_{2}^{J}+\chi_{4}^{J}+u \chi_{2}\right) q^{8}+\mathcal{O}\left(q^{\frac{17}{2}}\right),
\end{aligned}
$$

and on the gravity side we have

$$
\mathcal{I}_{X, 0}^{\mathrm{D} 3}=\cdots(\text { identical terms }) \cdots+u^{2}\left(-\chi_{2}^{J}+\chi_{4}^{J}\right) q^{8}+\mathcal{O}\left(q^{9}\right) .
$$

These are consistent with the third equation in (5.16).

\subsection{The other $\mathbb{C}^{3} / \mathbb{Z}_{7}$}

There is the other $\mathbb{Z}_{7}$ orbifold different from the one studied in the last subsection. Its orbifold group $\mathbb{Z}_{7}$ is generated by $\operatorname{diag}\left(\omega_{7}, \omega_{7}^{2}, \omega_{7}^{4}\right)$ and the dual group $\Gamma$ is characterized by the relations

$$
w_{X}^{2}=w_{Y}, \quad w_{Y}^{2}=w_{Z}, \quad w_{Z}^{2}=w_{X}, \quad w_{X} w_{Y} w_{Z}=e .
$$

The corresponding toric diagram and the quiver diagram are shown in figure 5. Among the seven sectors specified by $w=w_{X}^{k}(k=0,1,2, \ldots, 6)$ we are interested in one mesonic sector $w=e$ and three baryonic sectors $w=w_{X}, w=w_{Y}=w_{X}^{2}$, and $w=w_{Z}=w_{X}^{4}$ (see 


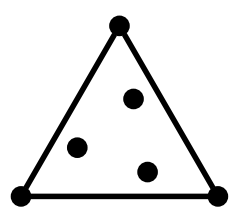

(a)

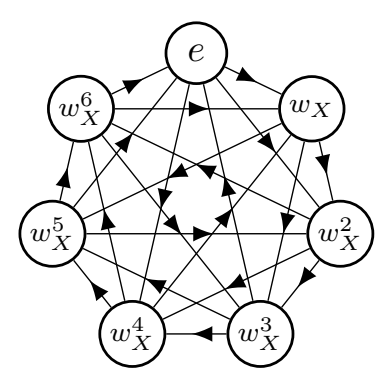

(b)

Figure 5. The toric diagram of $\mathbb{C}^{3} / \mathbb{Z}_{7}$ (a) and the corresponding quiver diagram (b) are shown.

\begin{tabular}{|ccc|}
\hline$w$ & $\mathcal{O}\left(q^{0}\right)$ and $\mathcal{O}\left(q^{N}\right)$ & higher order \\
\hline$e$ & 1 & $X Y Z, \ldots$ \\
$w_{X}$ & $X$ & $Z^{2}, \ldots$ \\
$w_{X}^{2}$ & $Y$ & $X^{2}, \ldots$ \\
$w_{X}^{3}$ & & $X Y, \ldots$ \\
$w_{X}^{4}$ & $Z$ & $Y^{2}, \ldots$ \\
$w_{X}^{5}$ & & $X Z, \ldots$ \\
$w_{X}^{6}$ & & $Y Z, \ldots$ \\
\hline
\end{tabular}

Table 4. Wrapping numbers $w \in \Gamma$ and the corresponding monomials $F\left(X_{I}\right)$ are shown for the orbifold $\mathcal{X}=\mathbb{C}^{3} / \mathbb{Z}_{7}$. The shaded rows are irrelevant to the leading corrections.

table 4). The expected relations for these sectors are

$$
\begin{aligned}
& \mathcal{I}_{0}^{\text {gauge }}=\mathcal{I}^{\text {grav }}+\mathcal{O}\left(q^{3 N}\right), \\
& \frac{\mathcal{I}_{\boldsymbol{b}_{e}-\boldsymbol{b}_{w_{X}}}^{\text {gauge }}}{\mathcal{I}_{0}^{\text {gauge }}}=\mathcal{I}_{X, 0}^{\mathrm{D} 3}+\mathcal{O}\left(q^{2 N}\right), \\
& \mathcal{I}_{\boldsymbol{b}_{e}-\boldsymbol{b}_{w_{X}^{2}}}^{\text {gauge }} \\
& \frac{\mathcal{I}_{0}^{\text {gauge }}}{\mathrm{D} 3}+\mathcal{O}\left(q^{2 N}\right), \\
& \frac{\mathcal{I}_{\boldsymbol{b}_{e}-\boldsymbol{b}_{w_{X}^{4}}}^{\text {gauge }}}{\mathcal{I}_{0}^{\text {gauge }}}=\mathcal{I}_{Z, 0}^{\mathrm{D} 3}+\mathcal{O}\left(q^{2 N}\right) .
\end{aligned}
$$

Let us confirm these equations for $N=2$.

The calculation on the gauge theory side gives the following index for the mesonic sector $w=e$ :

$$
\mathcal{I}_{0}^{\text {gauge }}=1-14 q^{6}+\mathcal{O}\left(q^{7}\right) .
$$

On the gravity side we have the following Kaluza-Klein index.

$$
\mathcal{I}^{\text {grav }}=1+0 q^{6}+\mathcal{O}\left(q^{7}\right) .
$$

These two are consistent with the first relation in (5.24). 
The latter three equations in (5.24) for the baryonic sectors are related by cyclic permutations of $X, Y$, and $Z$, and we explicitly check only the second in (5.24). On the gauge theory side the index is calculated as

$$
\begin{aligned}
\frac{\mathcal{I}_{\boldsymbol{b}_{e}-\boldsymbol{b}_{w_{X}}}^{\text {gauge }}}{\mathcal{I}_{0}^{\text {gauge }}}= & u^{2} q^{2}+\frac{u}{v^{2}} q^{3}+u^{2} \chi_{1}^{J} q^{\frac{7}{2}}+\left(\frac{v}{u}+\frac{1}{v^{4}}\right) q^{4} \\
& +\left(u^{2} \chi_{2}^{J}-u^{2}+\frac{1}{u^{2} v}+\frac{v^{4}}{u^{3}}\right) q^{5}+\mathcal{O}\left(q^{\frac{11}{2}}\right),
\end{aligned}
$$

and on the gravity side we obtain

$$
\mathcal{I}_{X, 0}^{\mathrm{D} 3}=\cdots(\text { identical terms }) \cdots+\left(u^{2} \chi_{2}^{J}-u^{2}+\frac{1}{u^{2} v}+\frac{v^{4}}{u^{3}}+\frac{1}{u v^{6}}\right) q^{5}+\mathcal{O}\left(q^{6}\right) .
$$

These two results are consistent with the second relation in (5.24).

$5.3 \mathbb{C} \times \mathbb{C}^{2} / \mathbb{Z}_{n}(n=2,3,4, \ldots)$

Let us consider the series of orbifolds $\mathcal{X}=\mathbb{C} \times \mathbb{C} / \mathbb{Z}_{n}(n=2,3,4, \ldots)$. We assume that $\mathbb{Z}_{n}$ acts on $Y$ and $Z$ as

$$
(X, Y, Z) \rightarrow\left(X, \omega_{n} Y, \omega_{n}^{-1} Z\right)
$$

The dual group $\Gamma$ is characterized by the relations

$$
w_{X}=w_{Y} w_{Z}=w_{Y}^{n}=e .
$$

If $n=2$, the relation $w_{Y}=w_{Z}$ holds and the system has an $\mathrm{SU}(2)$ flavor symmetry while if $n \geq 3 w_{Y} \neq w_{Z}$ and there is no continuous flavor symmetry. An important feature of these orbifolds is the existence of the fixed locus $Y=Z=0$, and we need to take account of the contribution of the tensor multiplets.

Although these systems preserve $\mathcal{N}=2$ supersymmetry the indices we calculate in this section are not $\mathcal{N}=2$ indices because of the difference in the treatment of the trace parts in the $\mathcal{N}=1$ vector multiplets and adjoint chiral multiplets. We include the trace contribution for the adjoint chiral multiplets while we do not for the vector multiplets.

\subsection{1 $\mathbb{C} \times \mathbb{C}^{2} / \mathbb{Z}_{2}$}

Let us first consider $n=2$ case: $\mathbb{C} \times \mathbb{C} / \mathbb{Z}_{2}$. The toric diagram and the quiver diagram are shown in figure 6 . There are two sectors specified by $w=e$ and $w=w_{Y}$ (table 5 ). The expected relations are

$$
\begin{aligned}
\mathcal{I}_{0}^{\text {gauge }} & =\mathcal{I}^{\text {grav }} \mathcal{I}_{X}^{\text {tensor }}\left(1+2 \mathcal{I}_{X, 0}^{\text {D3 }}\right)+\mathcal{O}\left(q^{2 N}\right), \\
\frac{\mathcal{I}_{\boldsymbol{b}_{e}-\boldsymbol{b}_{w_{Y}}}^{\text {gage }}}{\mathcal{I}_{0}^{\text {gauge }}} & =\mathcal{I}_{Y, 0}^{\text {D3 }}+\mathcal{I}_{Z, 0}^{\text {D3 }}+\mathcal{O}\left(q^{2 N}\right) .
\end{aligned}
$$




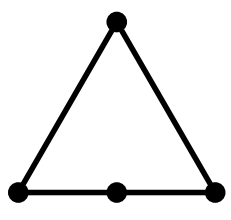

(a)

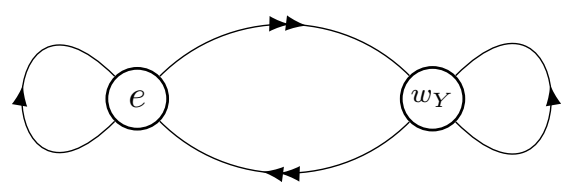

(b)

Figure 6. The toric diagram of $\mathbb{C} \times \mathbb{C}^{2} / \mathbb{Z}_{2}$ (a) and the corresponding quiver diagram (b) are shown.

\begin{tabular}{|ccc|}
\hline$w$ & $\mathcal{O}\left(q^{0}\right)$ and $\mathcal{O}\left(q^{N}\right)$ & higher order \\
\hline$e$ & $1, X$ & $X^{2}, \ldots$ \\
$w_{Y}$ & $Y, Z$ & $X Y, \ldots$ \\
\hline
\end{tabular}

Table 5. Wrapping numbers $w \in \Gamma$ and the corresponding monomials $F\left(X_{I}\right)$ are shown for the orbifold $\mathcal{X}=\mathbb{C} \times \mathbb{C}^{2} / \mathbb{Z}_{2}$.

We can numerically confirm the relations in (5.31) for $N=2$. For the mesonic sector with $w=e$ the calculation on the gauge theory side gives

$$
\begin{aligned}
\mathcal{I}_{0}^{\text {gauge }}= & 1+2 u q+\left(-\frac{1}{u}+5 u^{2}+\chi_{2}\right) q^{2}+\left(-2+8 u^{3}+2 u \chi_{2}\right) q^{3}+2 u^{2} \chi_{1}^{J} q^{\frac{7}{2}} \\
& +\left(\frac{1}{u^{2}}-7 u+14 u^{4}-\frac{2}{u} \chi_{2}+3 u^{2} \chi_{2}+2 \chi_{4}\right) q^{4}+\left(4 u^{3} \chi_{1}^{J}+2 u \chi_{1}^{J} \chi_{2}\right) q^{\frac{9}{2}} \\
& +\left(-14 u^{2}+20 u^{5}+2 u^{2} \chi_{2}^{J}-6 \chi_{2}+4 u^{3} \chi_{2}+2 u \chi_{4}\right) q^{5} \\
& +\left(\frac{2}{u^{2}} \chi_{1}^{J}-2 u \chi_{1}^{J}+10 u^{4} \chi_{1}^{J}+6 u^{2} \chi_{1}^{J} \chi_{2}+2 \chi_{1}^{J} \chi_{4}\right) q^{\frac{11}{2}} \\
& +\left(-\frac{1}{u^{3}}-25 u^{3}+30 u^{6}+4 u^{3} \chi_{2}^{J}+\frac{1}{u^{2}} \chi_{2}-13 u \chi_{2}+5 u^{4} \chi_{2}-\frac{2}{u} \chi_{4}\right. \\
& \left.\quad+3 u^{2} \chi_{4}+2 \chi_{6}\right) q^{6}+\mathcal{O}\left(q^{\frac{13}{2}}\right) .
\end{aligned}
$$

On the gravity side we obtain

$$
\begin{aligned}
\mathcal{I}^{\text {grav }} \mathcal{I}_{X}^{\text {tensor }}\left(1+2 \mathcal{I}_{X, 0}^{\text {D3 }}\right)= & \cdots(\text { identical terms }) \cdots \\
& +\left(-1-\frac{4}{u^{3}}-28 u^{3}+29 u^{6}+4 u^{3} \chi_{2}^{J}-16 u \chi_{2}+4 u^{4} \chi_{2}-\frac{5}{u} \chi_{4}\right. \\
& \left.\quad+2 u^{2} \chi_{4}+\chi_{6}\right) q^{6}+\mathcal{O}\left(q^{\frac{13}{2}}\right) .
\end{aligned}
$$

These results are consistent with the first equation in (5.31).

For the baryonic sector with $w=w_{Y}$ the localization on the gauge theory side gives

$$
\begin{aligned}
\frac{\mathcal{I}_{\boldsymbol{b}_{e}-\boldsymbol{b}_{w_{Y}}}^{\text {gauge }}}{\mathcal{I}_{0}^{\text {gauge }}}= & \chi_{2} q^{2}+\left(1-u \chi_{2}\right) q^{3}+\left(-\frac{1}{u} \chi_{1}^{J}+\chi_{1}^{J} \chi_{2}\right) q^{\frac{7}{2}}+\left(-\frac{2}{u^{2}}-u-u^{2} \chi_{2}\right) q^{4} \\
& +2 \chi_{1}^{J} q^{\frac{9}{2}}+\left(-u^{2}-\frac{1}{u} \chi_{2}^{J}-2 \chi_{2}+u^{3} \chi_{2}+\chi_{2}^{J} \chi_{2}\right) q^{5}+\mathcal{O}\left(q^{\frac{11}{2}}\right),
\end{aligned}
$$




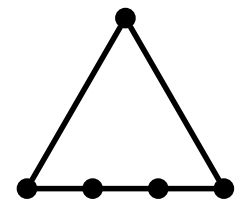

(a)

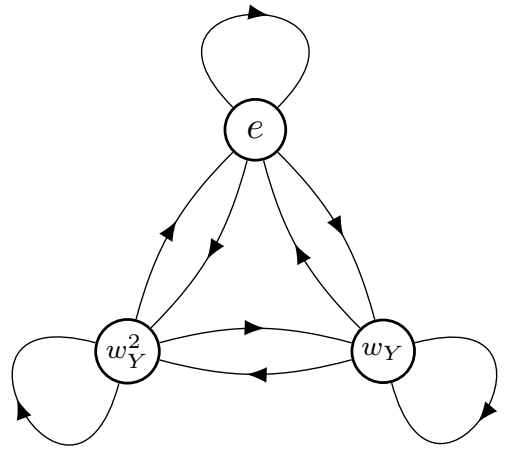

(b)

Figure 7. The toric diagram of $\mathbb{C} \times \mathbb{C}^{2} / \mathbb{Z}_{3}$ (a) and the corresponding quiver diagram (b) are shown.

\begin{tabular}{|ccc|}
\hline$w$ & $\mathcal{O}\left(q^{0}\right)$ and $\mathcal{O}\left(q^{N}\right)$ & higher order \\
\hline$e$ & $1, X$ & $X^{2}, \ldots$ \\
$w_{Y}$ & $Y$ & $X Y, \ldots$ \\
$w_{Y}^{2}$ & $Z$ & $X Z, \ldots$ \\
\hline
\end{tabular}

Table 6. Wrapping numbers $w \in \Gamma$ and the corresponding monomials $F\left(X_{I}\right)$ are shown for the orbifold $\mathcal{X}=\mathbb{C} \times \mathbb{C}^{2} / \mathbb{Z}_{3}$.

and the analysis on the gravity side gives

$$
\begin{aligned}
\mathcal{I}_{Y, 0}^{\mathrm{D} 3}+\mathcal{I}_{Z, 0}^{\mathrm{D} 3}= & \cdots(\text { identical terms }) \cdots \\
& +\left(-u^{2}-\frac{1}{u} \chi_{2}^{J}-2 \chi_{2}-u^{3} \chi_{2}+\chi_{2}^{J} \chi_{2}\right) q^{5}+\mathcal{O}\left(q^{\frac{11}{2}}\right)
\end{aligned}
$$

These two results are consistent with the second equation in (5.31).

\subsection{2 $\mathbb{C} \times \mathbb{C}^{2} / \mathbb{Z}_{3}$}

Let us consider the $n=3$ case with the orbifold $\mathcal{X}=\mathbb{C} \times \mathbb{C}^{2} / \mathbb{Z}_{3}$. The toric diagram and the quiver diagram are shown in figure 7 . There are three sectors specified by $w=e, w_{Y}, w_{Y}^{2}$. The sector with $w=e$ is the mesonic sector and the ones with $w=w_{Y}$ and $w=w_{Y}^{2}$ are baryonic sectors. See table 6 .

The expected relations are

$$
\begin{aligned}
\mathcal{I}_{0}^{\text {gauge }} & =\mathcal{I}^{\text {grav }}\left(\mathcal{I}_{X}^{\text {tensor }}\right)^{2}\left(1+3 \mathcal{I}_{X, 0}^{\mathrm{D} 3}\right)+\mathcal{O}\left(q^{2 N}\right), \\
\frac{\mathcal{I}_{\boldsymbol{b}_{e}-\boldsymbol{b}_{w_{Y}}}^{\text {gauge }}}{\mathcal{I}_{0}^{\text {gauge }}} & =\mathcal{I}_{Y, 0}^{\mathrm{D} 3}+\mathcal{O}\left(q^{2 N}\right), \\
\mathcal{I}_{\boldsymbol{b}_{e}-\boldsymbol{b}_{w_{Y}^{2}}}^{\text {gauge }} & =\mathcal{I}_{Z, 0}^{\mathrm{D} 3}+\mathcal{O}\left(q^{2 N}\right) . \\
\mathcal{I}_{0}^{\text {gauge }} &
\end{aligned}
$$


We numerically confirm these relations for $N=2$. For the mesonic sector the calculation on the gauge theory side gives

$$
\begin{aligned}
\mathcal{I}_{0}^{\text {gauge }}= & +3 u q+9 u^{2} q^{2}+\left(19 u^{3}+\frac{1}{v^{3}}+\frac{v^{3}}{u^{3}}\right) q^{3}+3 u^{2} \chi_{1}^{J} q^{\frac{7}{2}} \\
& +\left(-6 u+39 u^{4}+\frac{3 u}{v^{3}}+\frac{3 v^{3}}{u^{2}}\right) q^{4}+\left(3 \chi_{1}^{J}+9 u^{3} \chi_{1}^{J}\right) q^{\frac{9}{2}} \\
+ & \left(-\frac{6}{u}-21 u^{2}+69 u^{5}+\frac{6 u^{2}}{v^{3}}+\frac{6 v^{3}}{u}+3 u^{2} \chi_{2}^{J}\right) q^{5} \\
+ & \left(\frac{3}{u^{2}} \chi_{1}^{J}+9 u \chi_{1}^{J}+27 u^{4} \chi_{1}^{J}+\frac{3 u}{v^{3}} \chi_{1}^{J}+\frac{3 v^{3}}{u^{2}} \chi_{1}^{J}\right) q^{\frac{11}{2}} \\
+ & \left(-21+\frac{1}{u^{3}}-57 u^{3}+119 u^{6}+\frac{2}{v^{6}}-\frac{6}{v^{3}}+\frac{10 u^{3}}{v^{3}}+10 v^{3}-\frac{6 v^{3}}{u^{3}}\right. \\
& \left.\quad+\frac{2 v^{6}}{u^{6}}+9 u^{3} \chi_{2}^{J}\right) q^{6}+\mathcal{O}\left(q^{\frac{13}{2}}\right) .
\end{aligned}
$$

On the gravity side we obtain

$$
\begin{gathered}
\mathcal{I}^{\text {grav }}\left(\mathcal{I}^{\text {tensor }}\right)^{2}\left(1+3 \mathcal{I}_{X=0}^{\mathrm{D} 3}\right)=\cdots(\text { identical terms }) \cdots \\
+\left(-27-\frac{5}{u^{3}}-63 u^{3}+116 u^{6}+9 u^{3} \chi_{2}^{J}+\frac{6}{u} \chi_{1}-10 u^{2} \chi_{1}-6 \chi_{3}+10 u^{3} \chi_{3}\right. \\
\left.\quad-\frac{2}{u} \chi_{4}+2 \chi_{6}\right) q^{6}+\mathcal{O}\left(q^{\frac{13}{2}}\right)
\end{gathered}
$$

These two results are consistent with the first equation in (5.36).

Two baryonic sectors with $w=w_{Y}$ and $w=w_{Y}^{2}$ are related by the Weyl reflection swapping $Y$ and $Z$, and hence we check only the relation for $w=w_{Y}$ explicitly. The localization on the gauge theory side gives

$$
\begin{aligned}
\frac{\mathcal{I}_{\boldsymbol{b}_{e}-\boldsymbol{b}_{w_{Y}}}^{\text {gauge }}}{\mathcal{I}_{0}^{\text {gauge }}}= & \frac{v^{2}}{u^{2}} q^{2}+\left(\frac{1}{u v}-\frac{v^{2}}{u}\right) q^{3}+\frac{v^{2}}{u^{2}} \chi_{1}^{J} q^{\frac{7}{2}}+\left(\frac{1}{v^{4}}-v^{2}\right) q^{4} \\
& +\left(-\frac{2 u}{v^{4}}-\frac{1}{u^{2} v}-\frac{3 u}{v}-\frac{v^{2}}{u^{2}}+u v^{2}+\frac{v^{2}}{u^{2}} \chi_{2}^{J}\right) q^{5}+\mathcal{O}\left(q^{\frac{11}{2}}\right)
\end{aligned}
$$

and on the gravity side we obtain

$$
\begin{aligned}
\mathcal{I}_{Y, 0}^{\mathrm{D} 3}= & \cdots(\text { identical terms }) \cdots \\
& +\left(\frac{u}{v^{7}}-\frac{1}{u^{2} v}-\frac{u}{v}-\frac{v^{2}}{u^{2}}+\frac{v^{2}}{u^{2}} \chi_{2}^{J}\right) q^{5}+\mathcal{O}\left(q^{\frac{11}{2}}\right) .
\end{aligned}
$$

These results confirm the second equation in (5.36).

\section{$5.4 \mathbb{C}^{3} / \mathbb{Z}_{4}$}

Let us consider the orbifold group $\mathbb{Z}_{4}$ generated by $\operatorname{diag}\left(\omega_{4}^{-2}, \omega_{4}, \omega_{4}\right)$. The toric diagram and the quiver diagram are shown in figure 8 . 


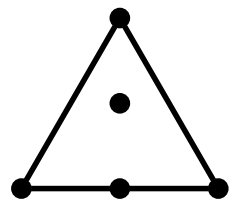

(a)

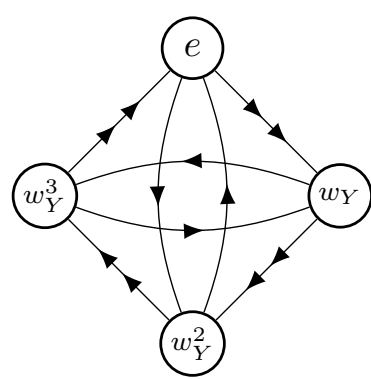

(b)

Figure 8. The toric diagram of $\mathbb{C}^{3} / \mathbb{Z}_{4}$ (a) and the corresponding quiver diagram (b) are shown.

\begin{tabular}{|ccc|}
\hline$w$ & $\mathcal{O}\left(q^{0}\right)$ and $\mathcal{O}\left(q^{N}\right)$ & higher order \\
\hline$e$ & 1 & $X^{2}, \ldots$ \\
$w_{Y}$ & $Y, Z$ & $X^{2} Y, \ldots$ \\
$w_{Y}^{2}$ & $X$ & $Y Z, \ldots$ \\
$w_{Y}^{3}$ & & $X Y, \ldots$ \\
\hline
\end{tabular}

Table 7. Wrapping numbers $w \in \Gamma$ and the corresponding monomials $F\left(X_{I}\right)$ are shown for the orbifold $\mathcal{X}=\mathbb{C}^{3} / \mathbb{Z}_{4}$. The shaded row is irrelevant to the leading corrections.

The dual group $\Gamma=\mathbb{Z}_{4}$ is characterized by the relations

$$
w_{Y}=w_{Z}, \quad w_{Y}^{2}=w_{X}, \quad w_{X}^{2}=e .
$$

There are four sectors specified by $w=w_{Y}^{k}(k=0,1,2,3)$ (table 7).

The expected relations are

$$
\begin{aligned}
\mathcal{I}^{\text {gauge }} & =\mathcal{I}^{\text {grav }} \mathcal{I}_{X}^{\text {tensor }}+\mathcal{O}\left(q^{2 N}\right), \\
\frac{\mathcal{I}_{\boldsymbol{b}_{e}-\boldsymbol{b}_{w_{Y}}}^{\text {gauge }}}{\mathcal{I}_{0}^{\text {gauge }}} & =\mathcal{I}_{Y, 0}^{\mathrm{D} 3}+\mathcal{I}_{Z, 0}^{\mathrm{D} 3}+\mathcal{O}\left(q^{3 N}\right), \\
\mathcal{I}_{\boldsymbol{b}_{e}-\boldsymbol{b}_{w_{Y}^{2}}}^{\text {gauge }} & =\mathcal{I}_{X, 0}^{\mathrm{D} 3}+\mathcal{O}\left(q^{2 N}\right) . \\
\mathcal{I}_{0}^{\text {gauge }} &
\end{aligned}
$$

Let us confirm these relations for $N=2$.

For the mesonic sector with $w=e$, the calculation on the gauge theory side gives

$$
\mathcal{I}_{0}^{\text {gauge }}=1+2 u^{2} q^{2}+\left(5 u^{4}-\frac{\chi_{2}}{u}+\chi_{4}\right) q^{4}-4 u^{2} q^{5}+\mathcal{O}\left(q^{\frac{11}{2}}\right) .
$$

On the gravity side we obtain

$$
\mathcal{I}^{\text {grav }} \mathcal{I}_{X}^{\text {tensor }}=1+2 u^{2} q^{2}+\left(5 u^{4}-\frac{\chi_{2}}{u}+\chi_{4}\right) q^{4}+0 q^{5}+\mathcal{O}\left(q^{6}\right) .
$$

These two results are consistent with the first relation in (5.42). 
For the baryonic sector with $w=w_{Y}$ the gauge theory calculation gives

$$
\begin{aligned}
\frac{\mathcal{I}_{\boldsymbol{b}_{e}-\boldsymbol{b}_{w_{Y}}}^{\text {gauge }}}{\mathcal{I}_{0}^{\text {gauge }}}= & \chi_{2} q^{2}+\chi_{1}^{J}\left(\chi_{2}-\frac{1}{u}\right) q^{\frac{7}{2}}+\left(u-\chi_{2} u^{2}\right) q^{4} \\
& +\left(\chi_{2}\left(\chi_{2}^{J}-1\right)-\frac{\chi_{2}^{J}}{u}-\frac{3}{u}\right) q^{5}+2 u \chi_{1}^{J} q^{\frac{11}{2}} \\
& -\left(\chi_{2}\left(u^{4}+\frac{1}{u^{2}}\right)+u^{3}-\frac{1}{u^{3}}\right) q^{6}+\chi_{3}^{J}\left(\chi_{2}-\frac{1}{u}\right) q^{\frac{13}{2}} \\
& +u\left(\chi_{2} u-3\right) q^{7}+\mathcal{O}\left(q^{\frac{15}{2}}\right)
\end{aligned}
$$

and the analysis on the gravity side gives

$$
\mathcal{I}_{Y, 0}^{\mathrm{D} 3}+\mathcal{I}_{Z, 0}^{\mathrm{D} 3}=\cdots(\text { identical terms }) \cdots-u\left(\chi_{2} u+1\right) q^{7}+\mathcal{O}\left(q^{\frac{15}{2}}\right) .
$$

These two results are consistent with the second relation in (5.42).

For the baryonic sector with $w=w_{Y}^{2}$ the calculation on the gauge theory side gives

$$
\begin{aligned}
\frac{\mathcal{I}_{\boldsymbol{b}_{e}-\boldsymbol{b}_{w_{Y}^{2}}}^{\text {gauge }}}{\mathcal{I}_{0}^{\text {gauge }}}= & u^{2} q^{2}+u \chi_{2} q^{3}+u^{2} \chi_{1}^{J} q^{\frac{7}{2}}+\left(\frac{1}{u^{2}}+\chi_{4}\right) q^{4} \\
& +u^{2}\left(\chi_{2}^{J}-1\right) q^{5}+\mathcal{O}\left(q^{\frac{11}{2}}\right),
\end{aligned}
$$

and the analysis on the gravity side gives

$$
\mathcal{I}_{X, 0}^{\mathrm{D} 3}=\cdots(\text { identical terms }) \cdots+\left(u^{2}\left(\chi_{2}^{J}-1\right)+\frac{1}{u} \chi_{6}+\frac{\chi_{2}}{u^{3}}\right) q^{5}+\mathcal{O}\left(q^{\frac{11}{2}}\right) .
$$

These results are consistent with the third relation in (5.42).

\section{$5.5 \mathbb{C}^{3} /\left(\mathbb{Z}_{2} \times \mathbb{Z}_{2}\right)$}

Finally, let us consider the orbifold with $\widetilde{\Gamma}=\mathbb{Z}_{2} \times \mathbb{Z}_{2}$ generated by $\operatorname{diag}(1,-1,-1)$ and $(-1,-1,1)$ The corresponding toric diagram and the quiver diagram are shown in figure 9. The dual group $\Gamma=\mathbb{Z}_{2} \times \mathbb{Z}_{2}$ is characterized by the following relations.

$$
w_{X}^{2}=w_{Y}^{2}=w_{Z}^{2}=w_{X} w_{Y} w_{Z}=e .
$$

There are four sectors specified by $w=e, w_{X}, w_{Y}, w_{Z}$ (table 8).

The expected relations are

$$
\begin{aligned}
\mathcal{I}_{0}^{\text {gauge }} & =\mathcal{I}^{\text {grav }} \mathcal{I}_{X}^{\text {tensor }} \mathcal{I}_{Y}^{\text {tensor }} \mathcal{I}_{Z}^{\text {tensor }}+\mathcal{O}\left(q^{2 N}\right), \\
\frac{\mathcal{I}_{\boldsymbol{b}_{e}-\boldsymbol{b}_{w_{X}}}^{\text {gauge }}}{\mathcal{I}_{0}^{\text {gauge }}} & =\mathcal{I}_{X, 0}^{\text {D3 }}+\mathcal{O}\left(q^{2 N}\right), \\
\frac{\mathcal{I}_{\boldsymbol{b}_{e}-\boldsymbol{b}_{w_{Y}}}^{\text {gauge }}}{\mathcal{I}_{0}^{\text {gauge }}} & =\mathcal{I}_{Y, 0}^{\mathrm{D} 3}+\mathcal{O}\left(q^{2 N}\right), \\
\frac{\mathcal{I}_{\boldsymbol{b}_{-}-\boldsymbol{b}_{w_{Z}}}^{\text {gaugg }}}{\mathcal{I}_{0}^{\text {gauge }}} & =\mathcal{I}_{Z, 0}^{\text {D3 }}+\mathcal{O}\left(q^{2 N}\right) .
\end{aligned}
$$

Let us conform these relations for $N=2$. 


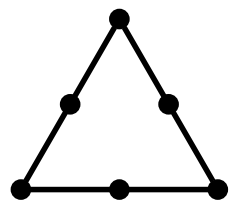

(a)

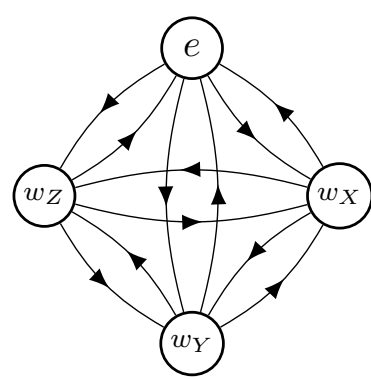

(b)

Figure 9. The toric diagram of $\mathbb{C}^{3} /\left(\mathbb{Z}_{2} \times \mathbb{Z}_{2}\right)$ (a) and the corresponding quiver diagram (b) are shown.

\begin{tabular}{|ccc|}
\hline$w$ & $\mathcal{O}\left(q^{0}\right)$ and $\mathcal{O}\left(q^{N}\right)$ & higher order \\
\hline$e$ & 1 & $X^{2}, \ldots$ \\
$w_{X}$ & $X$ & $Y Z, \ldots$ \\
$w_{Y}$ & $Y$ & $X Z, \ldots$ \\
$w_{Z}$ & $Z$ & $X Y, \ldots$ \\
\hline
\end{tabular}

Table 8. Wrapping numbers $w \in \Gamma$ and the corresponding monomials $F\left(X_{I}\right)$ are shown for the orbifold $\mathcal{X}=\mathbb{C}^{3} /\left(\mathbb{Z}_{2} \times \mathbb{Z}_{2}\right)$.

We first consider the mesonic sector with $w=e$. On the gauge theory side the index for $N=2$ is

$$
\begin{aligned}
\mathcal{I}_{0}^{\text {gauge }}= & 1+2\left(\chi_{(2,0)}-\chi_{(0,1)}\right) q^{2}+\left(4 \chi_{(0,2)}+\chi_{(1,0)}-5 \chi_{(2,1)}+5 \chi_{(4,0)}\right) q^{4} \\
& +4\left(\chi_{(0,1)}-\chi_{(2,0)}\right) q^{5}+\mathcal{O}\left(q^{\frac{11}{2}}\right)
\end{aligned}
$$

and on the gravity side we obtain

$$
\mathcal{I}^{\text {grav }} \mathcal{I}_{X}^{\text {tensor }} \mathcal{I}_{Y}^{\text {tensor }} \mathcal{I}_{Z}^{\text {tensor }}=\cdots \text { (identical terms) } \cdots+0 q^{5}+\mathcal{O}\left(q^{6}\right) .
$$

These results are consistent with the first relation in (5.50).

The three baryonic sectors are related among them by permutations among $X, Y$, and $Z$. We check explicitly the second equation in (5.50) associated with the baryonic sector with $w=w_{X}$. On the gauge theory side we obtain

$$
\begin{aligned}
\frac{\mathcal{I}_{\boldsymbol{b}_{e}-\boldsymbol{b}_{w_{X}}}^{\text {gauge }}}{\mathcal{I}_{0}^{\text {gauge }}}= & u^{2} q^{2}+q^{3}+u^{2} \chi_{1}^{J} q^{\frac{7}{2}}+\left(-\frac{u^{2}}{v^{2}}+\frac{1}{u^{2}}-v^{2}\right) q^{4} \\
& +\left(u^{2} \chi_{2}^{J}-u^{2}\right) q^{5}+\mathcal{O}\left(q^{\frac{11}{2}}\right)
\end{aligned}
$$

and on the gravity side we obtain

$$
\mathcal{I}_{X, 0}^{\mathrm{D} 3}=\cdots \text { (identical terms) } \cdots+\left(u^{2} \chi_{2}^{J}+\frac{1}{u^{4}}-u^{2}\right) q^{5}+\mathcal{O}\left(q^{6}\right) .
$$

These two results are consistent with the second relation in (5.50). 


\section{Summary and discussions}

We investigated the finite $N$ corrections to the superconformal index for quiver gauge theories realized on D3-branes put in abelian orbifolds $\mathcal{X}=\mathbb{C}^{3} / \widetilde{\Gamma}$. We focused on the leading corrections starting from $q^{N}$ and gave a prescription to calculate them on the gravity side as the contribution of wrapped D3-branes. Based on the analysis in [22] for S-fold theories we assumed that the corrections are reproduced as the contributions of D3-branes wrapped over three particular three-cycles, $X=0, Y=0$, and $Z=0$ in the internal space $\mathcal{Y}=\boldsymbol{S}^{5} / \widetilde{\Gamma}$. The D3-brane worldvolume of such a configuration is expressed by using a monomial function $F(X, Y, Z)$ as $F(X, Y, Z)=0$.

We established the relation between the D3-brane wrapping number and the baryonic charges. We gave a simple prescription to obtain baryonic charges from the function $F$ supplemented by holonomy variables. We compared indices calculated on the both sides of the AdS/CFT correspondence for each sector specified by the wrapping number of D3-branes.

In this paper we focused on the leading finite $N$ corrections, and did not study subleading corrections starting at $q^{k N}$ with $k \geq 2$ depending on the sector we considered. As far as we have checked for examples we found the complete agreement up to higher order terms of sub-leading corrections. This fact strongly suggests that our method is correct at least for configurations with a single D3-brane.

There are many open questions. An interesting and important task is the test of our method for the sub-leading corrections. In particular, analytic expressions for the exact Schur index is known for a few theories with finite $N[29,30]$ and it would be very nice if we could reproduce the complete index on the AdS side. Our assumption claims that they should be reproduced by D3-brane configurations including two or more wrapped D3-branes. Naively, the index for such configurations would be calculated as the index of non-abelian gauge theories realized on the coincident wrapped D3-branes. In addition, if the configuration contains branes wrapped on two different cycles open strings connecting them may contribute to the index. Further investigation is required to establish the method to calculate these contributions.

In the examples $\mathcal{X}=\mathbb{C} \times \mathbb{C} / \mathbb{Z}_{n}$ we found that D3-branes wrapped on a topologically trivial cycle give non-trivial contribution to the index. These branes are often referred to as giant gravitons, and such non-trivial contribution to the index had been also found in $\mathcal{N}=4$ SYM in [22]. On such branes a "tachyonic" degree of freedom lives and there exists a $q^{-1}$ term in the corresponding single-particle index. We formally deal with the plethystic exponential of this term by the relation

$$
\operatorname{Pexp}\left(q^{-1}\right)=-q \operatorname{Pexp}(q) .
$$

The negative sign appearing in this relation correctly reproduces the finite $N$ correction due to "the absence of giant gravitons." At present, unfortunately, we have no physical understanding of this mysterious agreement.

One potential way to justify our method rigorously is to use localization. Let $\mathcal{M}$ be the configuration space of D3-branes in the internal space $\mathcal{Y}$. The three configurations, 
$X=0, Y=0$, and $Z=0$ are fixed points of a rotation of $\mathcal{M}$ induced by an isometry of $\mathcal{Y}$. If we can extend $\mathcal{M}$ so that the path integral of the fields on D3-branes are treated as the integration over the extended space, and we can localize the integral to the points corresponding to the branes wrapped on the three cycles by using a localization theorem, then it would give a justification of the prescription we proposed in this paper.

In this paper we studied abelian orbifolds. There seems no obstacles to extend the method to non-abelian orbifolds and toric Sasaki-Einstein manifolds. In particular, for the latter class of manifolds the dual gauge theories are generically strongly coupled and the agreement of the superconformal index is highly non-trivial. There exists systematic prescription [31] to obtain the field contents of quiver gauge theory from the toric data of the dual internal space $\mathcal{Y}$, and it is in principle possible to calculate the index by using the localization formula. On the gravity side, as a naive analogy of the prescription for orbifolds we expect that the finite $N$ correction may be reproduced as the contribution of D3-branes wrapped on three-cycles in $\mathcal{Y}$. Although it may not be straightforward to calculate the single-particle indices for excitations on such three-cycles, once we obtain them we can easily test the proposal. We hope that we can come back to this problem in near future.

\section{Acknowledgments}

We would like to thank D. Yokoyama for valuable discussions and comments.

\section{A Results for $N=3$}

In this appendix we show the results for $N=3$. On the gravity side the calculation finishes instantly on a typical laptop computer. while it takes much longer time than the $N=2$ case to carry out the calculation on the gauge theory side. For some cases we could not obtain $\mathcal{I}_{w}^{\text {gauge }}$ up to sufficient order to find disagreement between $\mathcal{I}_{w}^{\text {AdS }}$ and $\mathcal{I}_{w}^{\text {gauge }}$. In the following we show the results for examples in which we could successfully finish the calculation up to the sufficient order.

\section{A.1 $\mathbb{C}^{3} / \mathbb{Z}_{3}$}

The relation (5.3) is confirmed for $N=3$ by comparing the following results.

$$
\begin{aligned}
& \mathcal{I}_{0}^{\text {gauge }}= 1+\left(1-\chi_{(1,1)}+\chi_{(3,0)}\right) q^{3} \\
&+\left(1+\chi_{(0,3)}-\chi_{(1,1)}+2 \chi_{(3,0)}-2 \chi_{(4,1)}+2 \chi_{(6,0)}\right) q^{6} \\
&+\left(2 \chi_{(0,3)}-2 \chi_{(1,1)}-2 \chi_{(1,4)}-3 \chi_{(2,2)}-\chi_{(3,0)}+2 \chi_{(3,3)}-2 \chi_{(4,1)}\right. \\
&\left.\quad-3 \chi_{(7,1)}+3 \chi_{(9,0)}-2\right) q^{9}+\mathcal{O}\left(q^{\frac{21}{2}}\right), \\
& \mathcal{I}^{\text {grav }}= \cdots(\text { identical terms }) \cdots \\
&+\left(1+2 \chi_{(0,3)}-2 \chi_{(1,1)}-2 \chi_{(1,4)}+2 \chi_{(3,0)}+2 \chi_{(3,3)}-2 \chi_{(4,1)}\right. \\
&\left.\quad+3 \chi_{(6,0)}-3 \chi_{(7,1)}+3 \chi_{(9,0)}\right) q^{9}+\mathcal{O}\left(q^{12}\right) .
\end{aligned}
$$


The relation (5.6) is confirmed for $N=3$ by comparing the following results.

$$
\begin{aligned}
& \frac{\mathcal{I}_{\boldsymbol{b}_{e}-\boldsymbol{b}_{w_{X}}}^{\text {gauge }}}{\mathcal{I}_{0}^{\text {gauge }}} \\
& =\chi_{(3,0)} q^{3}+\left(\chi_{(3,0)}-\chi_{(1,1)}\right) \chi_{1}^{J} q^{\frac{9}{2}} \\
& +\left(\left(-\chi_{(1,1)}+\chi_{(3,0)}+1\right) \chi_{2}^{J}-2 \chi_{(0,3)}-\chi_{(3,0)}\right) q^{6} \\
& +\left(\left(-\chi_{(0,3)}+2 \chi_{(1,1)}+\chi_{(2,2)}-\chi_{(3,0)}+1\right) \chi_{1}^{J}+\left(-\chi_{(1,1)}+\chi_{(3,0)}+1\right) \chi_{3}^{J}\right) q^{\frac{15}{2}} \\
& +\left(\left(2 \chi_{(1,1)}-2 \chi_{(3,0)}-1\right) \chi_{2}^{J}+\left(-\chi_{(1,1)}+\chi_{(3,0)}+1\right) \chi_{4}^{J}\right. \\
& \left.+2 \chi_{(0,3)}+\chi_{(1,4)}+\chi_{(2,2)}-2 \chi_{(3,0)}-\chi_{(4,1)}-\chi_{(5,2)}+5\right) q^{9} \\
& +\left(\left(\chi_{(0,3)}-4 \chi_{(1,1)}+\chi_{(1,4)}-3 \chi_{(2,2)}-3 \chi_{(3,0)}-2 \chi_{(3,3)}+\chi_{(4,1)}+\chi_{(6,0)}\right.\right. \\
& \left.\left.+\chi_{(7,1)}-1\right) \chi_{1}^{J}+\left(\chi_{(1,1)}-\chi_{(3,0)}-1\right)\left(\chi_{3}^{J}-\chi_{5}^{J}\right)\right) q^{\frac{21}{2}} \\
& +\left(\left(\chi_{(0,3)}-2 \chi_{(1,1)}-\chi_{(2,2)}+4 \chi_{(3,0)}-\chi_{(3,3)}+4 \chi_{(4,1)}+\chi_{(5,2)}-\chi_{(6,0)}-1\right) \chi_{2}^{J}\right. \\
& -\chi_{(1,1)} \chi_{6}^{J}+\left(\chi_{(1,1)}-\chi_{(3,0)}-1\right) \chi_{4}^{J}+\chi_{(3,0)} \chi_{6}^{J}+5 \chi_{(0,3)}+6 \chi_{(1,1)} \\
& +\chi_{(1,4)}+6 \chi_{(2,2)}+12 \chi_{(3,0)}+5 \chi_{(3,3)}+15 \chi_{(4,1)}+8 \chi_{(5,2)}+4 \chi_{(6,0)} \\
& \left.+\chi_{(6,3)}+\chi_{(7,1)}+\chi_{(9,0)}-\chi_{(10,1)}+\chi_{6}^{J}-10\right) q^{12}+\mathcal{O}\left(q^{\frac{25}{2}}\right), \\
& \mathcal{I}_{X, 0}^{\mathrm{D} 3}+\mathcal{I}_{Y, 0}^{\mathrm{D} 3}+\mathcal{I}_{Z, 0}^{\mathrm{D} 3} \\
& =\cdots \text { (identical terms }) \cdots \\
& +\left(\left(\chi_{(0,3)}-2 \chi_{(1,1)}-\chi_{(2,2)}+4 \chi_{(3,0)}-\chi_{(3,3)}+4 \chi_{(4,1)}+\chi_{(5,2)}\right.\right. \\
& \left.-\chi_{(6,0)}-1\right) \chi_{2}^{J}-\chi_{(1,1)} \chi_{6}^{J}+\left(\chi_{(1,1)}-\chi_{(3,0)}-1\right) \chi_{4}^{J}+\chi_{(3,0)} \chi_{6}^{J} \\
& +\chi_{(1,1)}-\chi_{(1,4)}+\chi_{(2,2)}+4 \chi_{(3,0)}+8 \chi_{(4,1)}+3 \chi_{(5,2)}+2 \chi_{(6,0)} \\
& \left.+\chi_{(6,3)}-\chi_{(7,1)}-\chi_{(9,0)}-\chi_{(10,1)}+\chi_{6}^{J}-5\right) q^{12}+\mathcal{O}\left(q^{\frac{25}{2}}\right) \text {. }
\end{aligned}
$$

\section{A.2 $\quad \mathbb{C}^{3} / \mathbb{Z}_{5}$}

The first relation in (5.9) is confirmed for $N=3$ by comparing the following results.

$$
\begin{aligned}
\mathcal{I}_{0}^{\text {gauge }} & =1+\left(u^{5}-\frac{\chi_{3}}{u}+\chi_{5}\right) q^{5}-5\left(2+u \chi_{2}+u^{2} \chi_{4}\right) q^{9}+\mathcal{O}\left(q^{\frac{19}{2}}\right), \\
\mathcal{I}^{\text {grav }} & =\cdots \text { (identical terms }) \cdots+0 q^{9}+\mathcal{O}\left(q^{10}\right) .
\end{aligned}
$$

The second relation in (5.9) is confirmed for $N=3$ by comparing the following results.

$$
\begin{aligned}
\frac{\mathcal{I}_{\boldsymbol{b}_{e}-\boldsymbol{b}_{w_{Y}}}^{\text {gauge }}}{\mathcal{I}_{0}^{\text {gauge }}=} & \chi_{3} q^{3}+u^{2} \chi_{2} q^{4}+\chi_{1}^{J}\left(\chi_{3}-\frac{\chi_{1}}{u}\right) q^{\frac{9}{2}}+u^{4} \chi_{1} q^{5}-u \chi_{1}^{J} q^{\frac{11}{2}} \\
& +\left(-\frac{\chi_{1} \chi_{2}^{J}}{u}+\chi_{3} \chi_{2}^{J}+u^{6}-\frac{\chi_{1}}{u}-\chi_{3}\right) q^{6}+\left(-2 u^{2} \chi_{2}-u\right) q^{7} \\
& +\left(2 u^{6} \chi_{1}^{J}+\frac{2 \chi_{1}^{J} \chi_{1}}{u}-\frac{\chi_{1} \chi_{3}^{J}}{u}-\chi_{3} \chi_{1}^{J}+\chi_{3}^{J} \chi_{3}\right) q^{\frac{15}{2}}+\mathcal{O}\left(q^{8}\right) \\
\mathcal{I}_{Y, 0}^{\mathrm{D} 3}+\mathcal{I}_{Z, 0}^{\mathrm{D} 3}= & \cdots(\text { identical terms }) \cdots \\
& +\left(u^{6} \chi_{1}^{J}+\frac{2 \chi_{1}^{J} \chi_{1}}{u}-\frac{\chi_{1} \chi_{3}^{J}}{u}-\chi_{3} \chi_{1}^{J}+\chi_{3}^{J} \chi_{3}\right) q^{\frac{15}{2}}+\mathcal{O}\left(q^{8}\right)
\end{aligned}
$$

For the third relation of (5.9) we could not finish the calculation on the gauge theory side. 


\section{A.3 $\mathbb{C} \times \mathbb{C}^{2} / \mathbb{Z}_{2}$}

The first relation in (5.31) is confirmed for $N=3$ by comparing the following results.

$$
\begin{aligned}
& \mathcal{I}_{0}^{\text {gauge }}=1+2 u q+\left(5 u^{2}-\frac{1}{u}+\chi_{2}\right) q^{2}+\left(10 u^{3}+2 u \chi_{2}-2\right) q^{3} \\
& +\left(18 u^{4}+5 u^{2} \chi_{2}+\frac{1}{u^{2}}-\frac{2 \chi_{2}}{u}-5 u+2 \chi_{4}\right) q^{4} \\
& +2 u^{3} \chi_{1}^{J} q^{\frac{9}{2}}+\left(30 u^{5}+8 u^{3} \chi_{2}-12 u^{2}+4 u \chi_{4}+\frac{2}{u}-4 \chi_{2}\right) q^{5} \\
& +\left(4 u^{4} \chi_{1}^{J}+2 u^{2} \chi_{2} \chi_{1}^{J}\right) q^{\frac{11}{2}} \\
& +\left(2 u^{3} \chi_{2}^{J}+49 u^{6}+14 u^{4} \chi_{2}-24 u^{3}-\frac{2}{u^{3}}+\frac{2 \chi_{2}}{u^{2}}+8 u^{2} \chi_{4}\right. \\
& \left.-12 u \chi_{2}-\frac{3 \chi_{4}}{u}+3 \chi_{6}+3\right) q^{6} \\
& +\left(10 u^{5} \chi_{1}^{J}+6 u^{3} \chi_{2} \chi_{1}^{J}-2 u^{2} \chi_{1}^{J}+2 u \chi_{4} \chi_{1}^{J}+\frac{2 \chi_{1}^{J}}{u}\right) q^{\frac{13}{2}} \\
& +\left(4 u^{4} \chi_{2}^{J}+74 u^{7}+20 u^{5} \chi_{2}-44 u^{4}+12 u^{3} \chi_{4}-26 u^{2} \chi_{2}\right. \\
& \left.-\frac{6}{u^{2}}+\frac{2 \chi_{2}}{u}+4 u \chi_{6}+4 u-8 \chi_{4}\right) q^{7} \\
& +\left(20 u^{6} \chi_{1}^{J}+14 u^{4} \chi_{2} \chi_{1}^{J}-6 u^{3} \chi_{1}^{J}+2 u^{3} \chi_{3}^{J}+\frac{2 \chi_{2} \chi_{1}^{J}}{u^{2}}\right. \\
& \left.+8 u^{2} \chi_{4} \chi_{1}^{J}+2 u \chi_{2} \chi_{1}^{J}+2 \chi_{6} \chi_{1}^{J}+6 \chi_{1}^{J}\right) q^{\frac{15}{2}} \\
& +\left(10 u^{5} \chi_{2}^{J}+2 u^{3} \chi_{2}^{J+1}-2 u^{2} \chi_{2}^{J}-2 \chi_{2}^{J} \chi_{2}+110 u^{8}+30 u^{6} \chi_{2}\right. \\
& -76 u^{5}+19 u^{4} \chi_{4}+\frac{3}{u^{4}}-47 u^{3} \chi_{2}-\frac{2 \chi_{2}}{u^{3}}+\frac{2 \chi_{4}}{u^{2}}+8 u^{2} \chi_{6} \\
& \left.+7 u^{2}-22 u \chi_{4}-\frac{4 \chi_{6}}{u}-\frac{13}{u}+\chi_{2}+4 \chi_{8}\right) q^{8}+\mathcal{O}\left(q^{\frac{17}{2}}\right),
\end{aligned}
$$

$\mathcal{I}^{\text {grav }} \mathcal{I}_{X}^{\text {tensor }}\left(1+2 \mathcal{I}_{X, 0}^{\mathrm{D} 3}\right)=\cdots($ identical terms $) \cdots$

$$
\begin{gathered}
+\left(10 u^{5} \chi_{2}^{J}+2 u^{3} \chi_{2}^{J+1}-2 u^{2} \chi_{2}^{J}-2 \chi_{2}^{J} \chi_{2}+109 u^{8}+29 u^{6} \chi_{2}\right. \\
-79 u^{5}+18 u^{4} \chi_{4}+\frac{2}{u^{4}}-50 u^{3} \chi_{2}-\frac{5 \chi_{2}}{u^{3}}+\frac{\chi_{4}}{u^{2}}+7 u^{2} \chi_{6} \\
\left.+6 u^{2}-25 u \chi_{4}-\frac{7 \chi_{6}}{u}-\frac{16}{u}+3 \chi_{8}\right) q^{8}+\mathcal{O}\left(q^{\frac{17}{2}}\right) .
\end{gathered}
$$


The second relation in (5.31) is confirmed for $N=3$ by comparing the following results.

$$
\begin{aligned}
\frac{\mathcal{I}_{\boldsymbol{b}_{e}-\boldsymbol{b}_{w_{Y}}}^{\text {gaage }}}{\mathcal{I}_{0}^{\text {gauge }}}= & \chi_{3} q^{3}+\left(\chi_{1}-u \chi_{3}\right) q^{4}+\left(\chi_{3} \chi_{1}^{J}-\frac{\chi_{1}^{J} \chi_{1}}{u}\right) q^{\frac{9}{2}}-\left(u^{2}\left(\chi_{3}\right)+\frac{\chi_{1}}{u^{2}}\right) q^{5} \\
& +\chi_{1}^{J} \chi_{1} q^{\frac{11}{2}}+\left(-\frac{\chi_{1} \chi_{2}^{J}}{u}+\chi_{3} \chi_{2}^{J}-2 u^{2} \chi_{1}-\frac{\chi_{1}}{u}-\chi_{3}\right) q^{6} \\
& +\left(-u^{2} \chi_{3} \chi_{1}^{J}+\frac{\chi_{1}^{J} \chi_{1}}{u^{2}}+3 u \chi_{1}^{J} \chi_{1}\right) q^{\frac{13}{2}} \\
& +\left(u^{4} \chi_{3}-u^{3} \chi_{1}-\frac{\chi_{3}}{u^{2}}-2 \chi_{1}\right) q^{7} \\
& +\left(-2 u^{3} \chi_{3} \chi_{1}^{J}+u^{2} \chi_{1}^{J} \chi_{1}-\frac{\chi_{1} \chi_{3}^{J}}{u}+\chi_{3}^{J} \chi_{3}\right) q^{\frac{15}{2}}+\mathcal{O}\left(q^{8}\right), \\
\mathcal{I}_{Y, 0}^{\mathrm{D} 3}+\mathcal{I}_{Z, 0}^{\mathrm{D} 3}= & \cdots(\text { identical terms }) \cdots+\left(u^{2} \chi_{1}^{J} \chi_{1}-\frac{\chi_{1} \chi_{3}^{J}}{u}+\chi_{3}^{J} \chi_{3}\right) q^{\frac{15}{2}} \\
& +\mathcal{O}\left(q^{8}\right) .
\end{aligned}
$$

\section{A.4 $\mathbb{C} \times \mathbb{C}^{2} / \mathbb{Z}_{3}$}

The first relation in (5.36) is confirmed for $N=3$ by comparing the following results.

$$
\begin{aligned}
& \mathcal{I}_{0}^{\text {gauge }} \\
& \begin{array}{l}
= \\
+
\end{array}+3 u q+9 u^{2} q^{2}+\left(-\frac{\chi_{1}}{u}+\chi_{3}+22 u_{3}^{3}\right) q^{3} \\
& +3 u\left(3 u \chi_{3}+32 \chi_{1}+48 u^{4}\right) q^{4}+3 u^{3} \chi_{1}^{J} q^{\frac{9}{2}} \\
& +\left(3 u^{3} \chi_{2}^{J}+19 \chi_{3} u^{3}-19 \chi_{1} u^{2}-\frac{2 \chi_{4}}{u}+2 \chi_{6}+182 u^{6}-21 u^{3}+\frac{1}{u^{3}}-6\right) q^{6} \\
& +3 \chi_{1}^{J}\left(\chi_{3} u^{2}-\chi_{1} u+9 u^{5}+3 u^{2}+\frac{1}{u}\right) q^{\frac{13}{2}} \\
& +\left(9 u^{4} \chi_{2}^{J}+\chi_{3}\left(39 u^{4}-6 u\right)+\chi_{1}\left(6-39 u^{3}\right)+6 \chi_{6} u\right. \\
& \left.\quad-6 \chi_{4}+324 u^{7}-63 u^{4}-\frac{3}{u^{2}}-27 u\right) q^{7} \\
& +3\left(\chi_{1}^{J}\left(\chi_{3}\left(4 u^{3}+1\right)-\chi_{1}\left(4 u^{2}+\frac{1}{u}\right)+22 u^{6}+8 u^{3}+5+\frac{1}{u^{3}}\right)\right. \\
& \left.\quad+u^{3} \chi_{3}^{J}\right) q^{\frac{15}{2}} \\
& +3\left(9 u^{5} \chi_{2}^{J}+5 \chi_{6} u^{2}-5 \chi_{4} u+\chi_{3}\left(23 u^{5}-9 u^{2}-\frac{1}{u}\right)+185 u^{8}\right. \\
& \left.\quad-51 u^{5}-24 u^{2}-\frac{6}{u}+\chi_{1}\left(-23 u^{4}+9 u+\frac{2}{u^{2}}\right)\right) q^{8}+\mathcal{O}\left(q^{\frac{17}{2}}\right)
\end{aligned}
$$




$$
\begin{aligned}
& \mathcal{I}^{\text {grav }}\left(\mathcal{I}_{X}^{\text {tensor }}\right)^{2}\left(1+3 \mathcal{I}_{X, 0}^{\mathrm{D} 3}\right) \\
& =\cdots(\text { identical terms }) \cdots \\
& \quad+3\left(9 u^{5} \chi_{2}^{J}+5 \chi_{6} u^{2}-5 \chi_{4} u+\chi_{3}\left(23 u^{5}-9 u^{2}-\frac{2}{u}\right)\right. \\
& \left.\quad+\chi_{1}\left(-23 u^{4}+9 u+\frac{1}{u^{2}}\right)+184 u^{8}-53 u^{5}-26 u^{2}-\frac{8}{u}-\frac{2}{u^{4}}\right) q^{8} \\
& \quad+\mathcal{O}\left(q^{\frac{17}{2}}\right) .
\end{aligned}
$$

The second and the third relations in (5.36) are related by the Weyl reflection. The second relation is confirmed for $N=3$ by comparing the following results.

$$
\begin{aligned}
\frac{\mathcal{I}_{\boldsymbol{b}_{e}-\boldsymbol{b}_{w_{Y}}}^{\text {gauge }}}{\mathcal{I}_{0}^{\text {gauge }}}= & \frac{v^{3}}{u^{3}} q^{3}+\left(\frac{1}{u^{2}}-\frac{v^{3}}{u^{2}}\right) q^{4}+\frac{v^{3}}{u^{3}} \chi_{1}^{J} q^{\frac{9}{2}}+\left(\frac{1}{u v^{3}}-\frac{v^{3}}{u}\right) q^{5} \\
& +\left(\frac{v^{3}}{u^{3}} \chi_{2}^{J}-\frac{v^{3}}{u^{3}}-\frac{1}{u^{3}}+\frac{1}{v^{6}}-1\right) q^{6}+\left(\frac{1}{u}-\frac{v^{3}}{u}\right) \chi_{1}^{J} q^{\frac{13}{2}} \\
& +\left(\frac{v^{3}}{u^{2}}-\frac{1}{u^{2}}-\frac{2 u}{v^{6}}+u v^{3}-\frac{2 u}{v^{3}}-3 u\right) q^{7}+\mathcal{O}\left(q^{\frac{15}{2}}\right), \\
\mathcal{I}_{Y, 0}^{\text {D3 }}= & \cdots(\text { identical terms }) \cdots \\
& +\left(\frac{u}{v^{9}}-u+\frac{v^{3}}{u^{2}}-\frac{1}{u^{2}}\right) q^{7}+\mathcal{O}\left(q^{\frac{15}{2}}\right) .
\end{aligned}
$$

\section{A.5 $\mathbb{C}^{3} / \mathbb{Z}_{4}$}

The first relation in (5.42) is confirmed for $N=3$ by comparing the following results.

$$
\begin{aligned}
\mathcal{I}_{0}^{\text {gauge }}= & 1+2 u^{2} q^{2}+\left(5 u^{4}-\frac{\chi_{2}}{u}+\chi_{4}\right) q^{4}+2 u\left(5 u^{5}-\chi_{2}+u \chi_{4}\right) q^{6} \\
& -4 u^{4} q^{7}+\mathcal{O}\left(q^{\frac{15}{2}}\right), \\
\mathcal{I}^{\text {grav }} \mathcal{I}_{X}^{\text {tensor }}= & \cdots \text { (identical terms }) \cdots+0 q^{7}+\mathcal{O}\left(q^{8}\right) .
\end{aligned}
$$

The second relation in (5.42) is confirmed for $N=3$ by comparing the following results.

$$
\begin{aligned}
\frac{\mathcal{I}_{\boldsymbol{b}_{e}-\boldsymbol{b}_{w_{Y}}}^{\text {gauge }}}{\mathcal{I}_{0}^{\text {gauge }}}= & \chi_{3} q^{3}+\chi_{1}^{J}\left(\chi_{3}-\frac{\chi_{1}}{u}\right) q^{\frac{9}{2}}+u\left(\chi_{1}-\chi_{3} u\right) q^{5} \\
& +\left(\chi_{3}\left(\chi_{2}^{J}-1\right)-\chi_{1}\left(\frac{\chi_{2}^{J}}{u}+\frac{1}{u}\right)\right) q^{6}+u \chi_{1} \chi_{1}^{J} q^{\frac{13}{2}}-\left(\chi_{3} u^{4}+\frac{\chi_{1}}{u^{3}}\right) q^{7} \\
& +\left(\chi_{1}\left(\frac{2 \chi_{1}^{J}}{u}-\frac{\chi_{3}^{J}}{u}\right)-\chi_{3}\left(\chi_{1}^{J}-\chi_{3}^{J}\right)\right) q^{\frac{15}{2}} \\
& +u\left(u \chi_{3}-4 \chi_{1}\right) q^{8}+\chi_{1}^{J}\left(\chi_{1}\left(3 u^{3}-\frac{1}{u^{3}}\right)-\chi_{3}\left(u^{4}-\frac{1}{u^{2}}\right)\right) q^{\frac{17}{2}} \\
& +\left(\chi_{1}\left(\frac{\chi_{2}^{J}}{u}-\frac{\chi_{4}^{J}}{u}-2 u^{5}+\frac{2}{u}\right)-\chi_{3}\left(\chi_{2}^{J}-\chi_{4}^{J}+3\right)\right) q^{9} \\
& +\left(\chi_{1}\left(\frac{2}{u^{3}}-3 u^{3}+\chi_{2}^{J} u^{3}\right)+\chi_{3}\left(\frac{1}{u^{2}}+u^{4}\right)-\frac{2}{u} \chi_{5}\right) q^{10}+\mathcal{O}\left(q^{\frac{21}{2}}\right), \\
\mathcal{I}_{Y, 0}^{\mathrm{D} 3}+\mathcal{I}_{Z, 0}^{\mathrm{D} 3}= & \cdots(\text { identical terms }) \cdots \\
& +\left(\chi_{1}\left(\frac{2}{u^{3}}-u^{3}+u^{3} \chi_{2}^{J}\right)+\frac{\chi_{3}}{u^{2}}-u^{4} \chi_{3}-\frac{2}{u} \chi_{5}\right) q^{10}+\mathcal{O}\left(q^{\frac{21}{2}}\right) .
\end{aligned}
$$


The third relation in (5.42) is confirmed for $N=3$ by comparing the following results.

$$
\begin{aligned}
\frac{\mathcal{I}_{\boldsymbol{b}_{e}-\boldsymbol{b}_{w_{Y}^{2}}}^{\text {gauge }}}{\mathcal{I}_{0}^{\text {gauge }}}= & u^{3} q^{3}+u^{2} \chi_{2} q^{4}+u^{3} \chi_{1}^{J} q^{\frac{9}{2}}+\left(u \chi_{4}+\frac{1}{u}\right) q^{5} \\
& +\left(u^{3}\left(\chi_{2}^{J}-1\right)+\frac{\chi_{2}}{u^{2}}+\chi_{6}\right) q^{6}-\chi_{2} \chi_{1}^{J} q^{\frac{13}{2}}-u^{2}\left(\chi_{4} u+\chi_{2}\right) q^{7} \\
& +\mathcal{O}\left(q^{\frac{15}{2}}\right), \\
\mathcal{I}_{X, 0}^{\mathrm{D} 3}= & \cdots(\text { identical terms }) \cdots \\
& +\left(-\chi_{2} u^{2}+\frac{\chi_{8}}{u}+\chi_{4}\left(\frac{1}{u^{3}}-u^{3}\right)+\frac{1}{u^{5}}\right) q^{7}+\mathcal{O}\left(q^{\frac{15}{2}}\right) .
\end{aligned}
$$

A.6 $\mathbb{C}^{3} /\left(\mathbb{Z}_{2} \times \mathbb{Z}_{2}\right)$

The first relation in (5.50) is confirmed for $N=3$ by comparing the following results.

$$
\begin{aligned}
\mathcal{I}_{0}^{\text {gauge }}= & 1+2\left(\chi_{(2,0)}-\chi_{(0,1)}\right) q^{2}+\left(4 \chi_{(0,2)}+\chi_{(1,0)}-5 \chi_{(2,1)}+5 \chi_{(4,0)}\right) q^{4} \\
& +\left(-10 \chi_{(0,3)}+10 \chi_{(2,2)}-10 \chi_{(4,1)}+10 \chi_{(6,0)}-2\right) q^{6} \\
& -4\left(\chi_{(0,2)}-\chi_{(2,1)}+\chi_{(4,0)}\right) q^{7}+\mathcal{O}\left(q^{8}\right)
\end{aligned}
$$

and on the gravity side we have

$$
\left.\mathcal{I}^{\text {grav }} \mathcal{I}_{X}^{\text {tensor }} \mathcal{I}_{Y}^{\text {tensor }} \mathcal{I}_{Z}^{\text {tensor }}=\cdots \text { (identical terms }\right) \cdots+0 q^{7}+\mathcal{O}\left(q^{8}\right)
$$

The second, the third, and the fourth relations in (5.50) are related by Weyl reflections among $X, Y$ and $Z$. The second relation in (5.50) is confirmed for $N=3$ by comparing the following results.

$$
\begin{aligned}
\frac{\mathcal{I}_{\boldsymbol{b}_{e}-\boldsymbol{b}_{w_{X}}}^{\text {gauge }}}{\mathcal{I}_{0}^{\text {gauge }}}= & u^{3} q^{3}+u q^{4}+u^{3} \chi_{1}^{J} q^{\frac{9}{2}}+\left(-\frac{u^{3}}{v^{2}}-u v^{2}+\frac{1}{u}\right) q^{5} \\
& +\left(u^{3} \chi_{2}^{J}-u^{3}+\frac{1}{u^{3}}\right) q^{6}+\left(-\frac{u^{3}}{v^{4}}-\frac{v^{4}}{u}-u\right) q^{7}+\mathcal{O}\left(q^{\frac{15}{2}}\right) \\
\mathcal{I}_{X, 0}^{\text {D3 }}= & \cdots(\text { identical terms }) \cdots+\left(\frac{1}{u^{5}}-\frac{u^{3}}{v^{4}}-\frac{v^{4}}{u}-u\right) q^{7}+\mathcal{O}\left(q^{\frac{15}{2}}\right)
\end{aligned}
$$

Open Access. This article is distributed under the terms of the Creative Commons Attribution License (CC-BY 4.0), which permits any use, distribution and reproduction in any medium, provided the original author(s) and source are credited.

\section{References}

[1] J.M. Maldacena, The Large $N$ limit of superconformal field theories and supergravity, Int. J. Theor. Phys. 38 (1999) 1113 [hep-th/9711200] [INSPIRE].

[2] E. Witten, Anti-de Sitter space and holography, Adv. Theor. Math. Phys. 2 (1998) 253 [hep-th/9802150] [INSPIRE].

[3] S.S. Gubser, I.R. Klebanov and A.M. Polyakov, Gauge theory correlators from noncritical string theory, Phys. Lett. B 428 (1998) 105 [hep-th/9802109] [INSPIRE]. 
[4] J. Kinney, J.M. Maldacena, S. Minwalla and S. Raju, An Index for 4 dimensional super conformal theories, Commun. Math. Phys. 275 (2007) 209 [hep-th/0510251] [INSPIRE].

[5] S. Kachru and E. Silverstein, 4-D conformal theories and strings on orbifolds, Phys. Rev. Lett. 80 (1998) 4855 [hep-th/9802183] [INSPIRE].

[6] A.E. Lawrence, N. Nekrasov and C. Vafa, On conformal field theories in four-dimensions, Nucl. Phys. B 533 (1998) 199 [hep-th/9803015] [INSPIRE].

[7] Y. Nakayama, Index for orbifold quiver gauge theories, Phys. Lett. B 636 (2006) 132 [hep-th/0512280] [INSPIRE].

[8] Y. Nakayama, Index for supergravity on $A d S_{5} \times T^{1,1}$ and conifold gauge theory, Nucl. Phys. B 755 (2006) 295 [hep-th/0602284] [INSPIRE].

[9] I.R. Klebanov and E. Witten, Superconformal field theory on three-branes at a Calabi-Yau singularity, Nucl. Phys. B 536 (1998) 199 [hep-th/9807080] [INSPIRE].

[10] A. Gadde, L. Rastelli, S.S. Razamat and W. Yan, On the Superconformal Index of $N=1$ IR Fixed Points: A Holographic Check, JHEP 03 (2011) 041 [arXiv: 1011.5278] [INSPIRE].

[11] E. Witten, Baryons and branes in anti-de Sitter space, JHEP 07 (1998) 006 [hep-th/9805112] [INSPIRE].

[12] S. Gukov, M. Rangamani and E. Witten, Dibaryons, strings and branes in AdS orbifold models, JHEP 12 (1998) 025 [hep-th/9811048] [INSPIRE].

[13] J. McGreevy, L. Susskind and N. Toumbas, Invasion of the giant gravitons from Anti-de Sitter space, JHEP 06 (2000) 008 [hep-th/0003075] [INSPIRE].

[14] S. Benvenuti, B. Feng, A. Hanany and Y.-H. He, Counting BPS Operators in Gauge Theories: Quivers, Syzygies and Plethystics, JHEP 11 (2007) 050 [hep-th/0608050] [INSPIRE].

[15] I. Biswas, D. Gaiotto, S. Lahiri and S. Minwalla, Supersymmetric states of $N=4$ Yang-Mills from giant gravitons, JHEP 12 (2007) 006 [hep-th/0606087] [INSPIRE].

[16] C.E. Beasley, BPS branes from baryons, JHEP 11 (2002) 015 [hep-th/0207125] [INSPIRE].

[17] A. Mikhailov, Giant gravitons from holomorphic surfaces, JHEP 11 (2000) 027 [hep-th/0010206] [INSPIRE].

[18] G. Mandal and N.V. Suryanarayana, Counting 1/8-BPS dual-giants, JHEP 03 (2007) 031 [hep-th/0606088] [INSPIRE].

[19] M.T. Grisaru, R.C. Myers and O. Tafjord, SUSY and goliath, JHEP 08 (2000) 040 [hep-th/0008015] [INSPIRE].

[20] A. Hashimoto, S. Hirano and N. Itzhaki, Large branes in AdS and their field theory dual, JHEP 08 (2000) 051 [hep-th/0008016] [INSPIRE].

[21] R. Arai, S. Fujiwara and Y. Imamura, BPS Partition Functions for S-folds, JHEP 03 (2019) 172 [arXiv: 1901.00023] [INSPIRE].

[22] R. Arai and Y. Imamura, Finite $N$ Corrections to the Superconformal Index of S-fold Theories, PTEP 2019 (2019) 083B04 [arXiv: 1904.09776] [INSPIRE].

[23] M.R. Douglas and G.W. Moore, D-branes, quivers and ALE instantons, hep-th/9603167 [INSPIRE]. 
[24] M.R. Douglas, B.R. Greene and D.R. Morrison, Orbifold resolution by D-branes, Nucl. Phys. B 506 (1997) 84 [hep-th/9704151] [INSPIRE].

[25] H.J. Kim, L.J. Romans and P. van Nieuwenhuizen, The Mass Spectrum of Chiral $N=2$ $D=10$ Supergravity on $S^{5}$, Phys. Rev. D 32 (1985) 389 [InSPIRE].

[26] M. Günaydin and N. Marcus, The Spectrum of the $S^{5}$ Compactification of the Chiral $N=2$, $D=10$ Supergravity and the Unitary Supermultiplets of U(2,2/4), Class. Quant. Grav. 2 (1985) L11 [INSPIRE].

[27] F.A. Dolan and H. Osborn, On short and semi-short representations for four-dimensional superconformal symmetry, Annals Phys. 307 (2003) 41 [hep-th/0209056] [INSPIRE].

[28] C. Cordova, T.T. Dumitrescu and K. Intriligator, Deformations of Superconformal Theories, JHEP 11 (2016) 135 [arXiv: 1602.01217] [INSPIRE].

[29] J. Bourdier, N. Drukker and J. Felix, The exact Schur index of $\mathcal{N}=4$ SYM, JHEP 11 (2015) 210 [arXiv: 1507.08659] [INSPIRE].

[30] J. Bourdier, N. Drukker and J. Felix, The $\mathcal{N}=2$ Schur index from free fermions, JHEP 01 (2016) 167 [arXiv: 1510.07041] [InSPIRE].

[31] A. Hanany and D. Vegh, Quivers, tilings, branes and rhombi, JHEP 10 (2007) 029 [hep-th/0511063] [INSPIRE]. 